\title{
Reference mirroring for control with impacts
}

\subsection{Introduction}

Control and state estimation of dynamical systems subject to impacts are relevant problems in several application areas, often related to the robotics field [BRO 99]. Impacts play a key role in several studies including hopping robots (see, e.g., [SCH 95]), walking robots (see, e.g., [MOR 09]) and juggling robots (see, e.g., [RON 06]). Several Lyapunov-based solutions to the stabilization and tracking problems of systems with impacts have been proposed in the past decade [BRO 04, LEI 08a, TOR 99], and several studies have been developed for the dual state-estimation problem [MEN 01c, MEN 01b, GAL 03]. Some of them address the problem via the larger class of complementarity Lagrangian systems (see [HEE 03] for a survey, and the overview in [MOR 10] which also generalizes and improves the results in [BOU 05, BRO 97]). Within this field, tracking in billiards is a relevant and representative example where much has been done using the model first proposed in [TOR 99]. See [GAL 08] and references therein. Several additional recent techniques addressing tracking control with impacts both from a theoretical and an experimental viewpoint are provided in the works [PAG 01, PAG 04, LEI 08b, MEN 01a, SEK 06] and references therein. See [MOR 10] for a more detailed overview.

One important obstruction to effective tracking and state observation laws for systems with impacts is that when stabilizing the error dynamics (associated with tracking or observation laws), classical continuous-time solutions that disregard the effects of the impacts may not work properly due to the undesired effects of impacts. We show in Section 8.2 that even a simple linear exponential tracking for a one dimensional system may produce an unstable error dynamics when applied to the impacting dynamics. An alternative example with a similar flavor and a formal analysis of instability has been also given in [FOR 11a]. Motivated by this obstruction and the goal of

Chapter written by F. Forni, A.R. TEEL and L. ZACCARIAN . 
recovering linear error dynamics, in our recent work [FOR 11b, FOR 11c, FOR 11a], we formulated the tracking and observation problems for a mass moving in a planar confined region as a hybrid stabilization problem for a suitable set. In particular, by casting the tracking or observation problems within the hybrid framework of [GOE 06, GOE 12, GOE 09], asymptotic tracking or estimation is written as an exponential stabilization problem for a closed set (or attractor) $\mathcal{A}$ where certain relevant sub-states are equal. The core ideas of [FOR 11b, FOR 11c, FOR 11a] have been later extended in [FOR 12] to the case of a mass moving into an $n$ dimensional half space. Similar ideas to those in [FOR 11b] were independently presented later as an application of the Lyapunov conditions in [BIE 12, BIE 13], with special emphasis on the one-dimensional case of a bouncing ball.

In this book chapter we illustrate the main ideas behind [FOR 11b, FOR 11c, FOR 11a, FOR 12] by emphasizing the fundamental intuition behind our approach, which allows to recover a classical (quasi-)linear control algorithm for tracking and state-estimation of point-masses evolving in an $n$-dimensional space in the presence of impacts. Given a controlled (or observer) system and a reference system with state vectors $x$ and $z$ respectively, we replace the usual feedback error $e=x-z$ by a generalized error $e=x-m(q, z)$. The logic variable $q$ is typically updated at each impact and keeps track of the (finite set of) impact phenomena $\mathcal{Q}:=\{1, \ldots, d\}$. The function $m(q, z)$ is an affine transformation on $z$ that ensures the non-increase of the error magnitude at impacts, as measured by a suitable quadratic error Lyapunov function. The introduction of the generalized error $e$ is driven by the suggestive idea that the effect on an impact on a boundary can be virtually inverted by using the boundary as a mirror. In this sense, the transformation $m(q, z)$ provides the control algorithm with a feedback measurement of a suitable mirrored reference motion "through" the mirror/boundary, with the goal of recovering the exponential decrease of the error along the trajectories of the (hybrid) closed-loop system.

In this chapter we illustrate the intuition above by addressing several examples where the loss of performance experienced with linear controllers is recovered by the introduction of the generalized error $e=x-m(q, z)$. We start our discussion with a one-dimensional system in Section 8.2, whose dynamics resembles that of a bouncing ball even though the engineering motivation behind this example is somewhat more intuitive. We then discuss a slightly more complicated example of a Newton cradle, using a model taken from the literature [MEN 02] and showing that similar ideas apply here too. We then move on to a much more general system where a mass is constrained to evolve in an equilateral triangular subset of the position subspace. The construction of the mirrored references $m(q, z)$ for this case becomes more involved and is illustrated graphically to preserve the intuitive style of the chapter. Finally, in Section 8.5 we address an apparently different estimation problem where there is no need to define mirrored references and the analysis then greatly simplifies. 
Each one of the discussed examples contains a hybrid formulation of the impacting dynamics together with the proof of a key property about the behavior of the generalized error $e=x-m(q, z)$ across impacts, which can be derived from the jump dynamics of the hybrid model. Then, a theorem states exponential convergence to zero of the generalized error and a proposition illustrates how one can draw suitable conclusions on the actual error $x-z$ from properties of the generalized error (this is not necessary for the last example where no mirrors are used). All the theorems stating exponential convergence are proven using the results in [TEE 12] and to better highlight the strong commonalities among the proofs (despite the very different nature of all the examples), the proofs of these theorems are gathered together in Section 8.6, where a Lyapunov reformulation of [TEE 12, Theorem 2] is also given to facilitate the analysis.

Notation: For any given vector $z \in \Re^{2 n}, z_{i}$ denotes the $i$ th component of $z, z_{p}$ (positions) denotes the sub-vector given by $z_{1}, \ldots, z_{n}$, and $z_{v}$ (velocities) denotes the sub-vector given by $z_{n+1}, \ldots, z_{2 n} . I_{n}$ is the identity matrix of dimension $n$. Given two matrices $A$ and $B$, their Kronecker product is denoted by $A \otimes B$.

\subsection{Hammering a surface}

The first example that we propose is a mechanical system with one degree of freedom experiencing impacts comprising a hammer hitting on a surface. This example clearly illustrates the possible dangers of blindly using linear tracking controllers whenever impacts are experienced. Similar results have been presented for a bouncing ball in [FOR 11b, FOR 11a]. The hammer formulation of the same phenomenon perhaps has a better engineering motivation.

\subsubsection{The reference hammer dynamics}

Let us consider the reference dynamics $\mathcal{Z}$ of a hammer impacting on a surface, as shown in Figure 8.32. The angular position of the hammer with respect to the horizontal line is represented by the state variable $z_{p}$, while its angular velocity is denoted by $z_{v}$. The hammer is driven towards the plane by the concurrent action of a spring, which exerts the torque $-k_{s} z_{p}$ (where $k_{s}>0$ is the spring characteristic constant) at the hinge, and of the gravity that, assuming for simplicity unit mass, generates the torque $-g \cos \left(z_{p}\right)$ at the hinge. The dynamics of the system is given by

$$
\ddot{z}_{p}=-k_{s} z_{p}-g \cos \left(z_{p}\right) \quad z \in C_{z}:=\left\{\left(z_{p}, z_{v}\right) \in \Re^{2}: z_{p} \in[0, \pi]\right\},
$$

where the closed set $C_{z} \subset \Re^{2}$ is instrumental to restricting the position $z_{1}$ of the hammer to the upper half plane of Figure 8.32. Indeed, solutions that would reach beyond those points are prematurely terminated by the fact that they cannot flow (nor jump). 


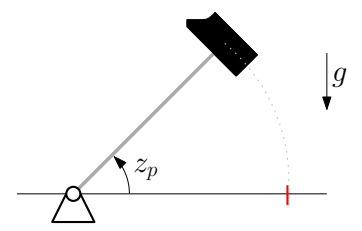

Figure 8.32: The reference hammer impacts on the horizontal plane.

Using the aggregate state $z:=\left[\begin{array}{ll}z_{p} & z_{v}\end{array}\right]^{T}$, a convenient formulation of the flow dynamics (8.250) is given by

$$
\dot{z}=\bar{A} z+\bar{B} \alpha_{z}(z) \quad z \in C_{z}
$$

where

$$
\bar{A}:=\left[\begin{array}{ll}
0 & 1 \\
0 & 0
\end{array}\right], \quad \bar{B}:=\left[\begin{array}{l}
0 \\
1
\end{array}\right], \quad \alpha_{z}(z):=-k_{s} z_{p}-g \cos \left(z_{p}\right) .
$$

which highlights the structure of a double integrator driven by (possibly nonlinear) state dependent acceleration terms.

At impacts, the sign of the speed $z_{v}$ is inverted, while the position $z_{p}$ remains constant. However, due to the convenient selection of the coordinate system where impacts occur only if $z_{p}=0$, we can write the impact (or jump) dynamics of the hammer as:

$z^{+}=\left[\begin{array}{c}z_{p} \\ -z_{v}\end{array}\right]=\left[\begin{array}{c}0 \\ -z_{v}\end{array}\right]=-z, \quad z \in D_{z}:=\left\{\left(z_{p}, z_{v}\right) \in \Re^{2}: z_{p}=0, z_{v} \leq 0\right\}$,

namely, similar to the well studied example of a bouncing ball (see, e.g., [GOE 09, Example 3]) the specific selection of the coordinate system simplifies the jump map.

The combination of the flow dynamics (8.251) and the jump dynamics (8.252) and the flow and jump sets $C_{z}, D_{z}$ provide a hybrid formulation of the reference hammer dynamics, according to the notation proposed in [GOE 12, GOE 09]. For dealing with this example and the rest of the examples treated in this chapter we will use results from this specific framework for describing hybrid dynamical systems. We don't recall here the essential definitions from [GOE 12, GOE 09]. The reader is referred to those works for an introduction of that framework or, alternatively, to the brief overview in [NEĨ1, Section II].

\subsubsection{Using dwell-time logic to avoid Zeno solutions}

Both the flow set $C_{z}$ and the jump set $D_{z}$ in the hybrid model (8.251), (8.252) are intentionally characterized as closed sets so that suitable regularity properties of the 
solutions set of our hybrid system are guaranteed (see, e.g, [GOE 12, Chapter 6]). Due to this reason, some solutions may exhibit an infinite number of jumps (namely a Zeno behavior) when starting from the initial condition with $z_{1}=0$ and $z_{2}=0$. To avoid this particular side effect of the adopted dynamical model, we embed in our dynamics a so-called average dwell time logic, as characterized in the appendix of [CAI 08] (see also [GOE 12, Example 2.15]) parametrized by a real $\rho>0$ and an integer $N \geq 1$ and satisfying the flow and jump equations:

$$
\begin{array}{llll}
\dot{\sigma} & \in[0, \rho], & & \sigma \in[0, N] \\
\sigma^{+}=\sigma-1, & & \sigma \in[1, N] .
\end{array}
$$

As formally proven in [CAI 08, Proposition 1.1], embedding the extra state $\sigma$ in the hybrid description and intersecting the sets $C_{z}$ and $D_{z}$ with the rules in (8.253), ensures that all solutions satisfy a persistent flow conditions, namely for each solution $\phi$ to the hybrid system and each pair $(s, i),(t, j)$ of consecutive hybrid times in its domain, the following holds:

$$
j-i \leq \rho(t-s)+N
$$

which clearly imposes an upper bound on the number of jumps that the solution can perform between two consequent ordinary times $t$ and $s$. Note that, as a special case, no solution can perform more than $N$ simultaneous jumps if its dynamics embeds the $\operatorname{logic}(8.253)$.

Throughout this chapter we will embed the dwell-time logic (8.253) in our models to remove the undesired Zeno side-effect of this specific formulation, and ensure that complete solutions have unbounded domain in the ordinary time direction $t$. Nevertheless, no restrictions will be imposed on the dwell-time parameters $(\rho, N)$ so that any reasonable behavior of the described impacting system will be captured and not terminated by the hybrid dynamics with average dwell-time regularization, as long as $\rho$ and $N$ are selected large enough. In particular, the arbitrariness of $\rho$ and $N$ in our solutions comes from the fact that nowhere in our synthesized stabilizers or observers there will be an explicit dependence of the parameters on the parameters $\rho$ and $N$ and the stated properties will hold for any such selection.

\subsubsection{The controlled hammer dynamics}

The reference hammer dynamics $(8.251),(8.252)$ can be well understood as a fictitious (or exogenous) dynamics specifying a desired motion for a real hammer, whose behavior should be tracked by the real hammer. The dynamics of the real hammer is the same as that of the reference hammer with the extra feature that a torque input $u$ is available at the hinge and that the beneficial effect of the (fictitious) spring acting on the reference dynamics is replaced by an undesired viscous friction effect affecting the real hammer flow dynamics. In particular, using the notation introduced in 
(8.251), the real, or controlled, hammer dynamics is described by the following flow dynamics:

$$
\dot{x}=\bar{A} x+\bar{B}\left(\alpha_{x}(x)+u\right) \quad x \in C_{x}:=\left\{\left(x_{p}, x_{v}\right) \in \Re^{2}: x_{p} \in[0, \pi]\right\},
$$

where $u$ is the control input to be used for reference tracking and where

$$
\alpha_{x}(x):=-k_{f} x_{v}-g \cos \left(x_{p}\right)
$$

with $k_{f}>0$ being the coefficient of the viscous friction clearly generating a dissipative action. Similar to before, the non-dissipative impact dynamics of the controlled hammer is given by

$$
x^{+}=-x \quad x \in D_{x}:=\left\{x \in \Re^{2}: x_{p}=0, x_{v} \leq 0\right\} .
$$

Summarizing the derivations of Sections 8.2.1-8.2.3, we can lump into a single hybrid dynamical system the equations of the reference and controlled hammer dynamics with average dwell time. In particular, we get:

$$
\left\{\begin{aligned}
\dot{z} & =\bar{A} z+\bar{B} \alpha_{z}(z) \\
\dot{x} & =\bar{A} x+\bar{B}\left(\alpha_{x}(x)+u\right) \quad(z, x, \sigma) \in C_{z} \times C_{x} \times[0, N] \\
\dot{\sigma} & \in[0, \rho]
\end{aligned}\right.
$$

Moreover an impact of the reference hammer is given by

$$
\left\{\begin{array}{l}
z^{+}=-z \\
x^{+}=x \\
\sigma^{+}=\sigma-1,
\end{array} \quad(z, x, \sigma) \in D_{z} \times\left(C_{x} \cup D_{x}\right) \times[1, N],\right.
$$

while impacts of the controlled hammer are described by

$$
\left\{\begin{array}{l}
z^{+}=z \\
x^{+}=-x \\
\sigma^{+}=\sigma-1,
\end{array} \quad(z, x, \sigma) \in\left(C_{z} \cup D_{z}\right) \times D_{x} \times[1, N] .\right.
$$

Note that a synchronous impact of the reference and controlled hammer is characterized by two consecutive jumps of the overall hybrid system given by either (8.257) followed by (8.258), or vice versa. This is allowed by the average dwell time logic as long as the (free) parameter $N$ is selected as $N \geq 2$. The control input $u$ is still unspecified in the dynamics (8.256)-(8.258). In the next two sections we will first discuss the unsuitability of a classical continuous-time tracking feedback selection for $u$ and then we will illustrate the properties of the proposed hybrid solution based on mirrored references. 


\subsubsection{Instability with standard feedback tracking}

The simplest control approach to the solution of the tracking problem is given by the compensation of the nonlinear gravity terms, and by the injection of a suitable linear error feedback in the control input $u$, to guarantee that the error dynamics $e=$ $x-z$ is exponentially converging to zero along flows. In particular, focusing on the flow equations (8.256), one may select

$$
u=\alpha_{z}(z)-\alpha_{x}(x)+\bar{K}(x-z),
$$

where $\bar{K}=\left[\begin{array}{ll}k_{p} & k_{v}\end{array}\right]$ is such that $\bar{A}_{c l}:=\bar{A}+\overline{B K}=\left[\begin{array}{cc}0 & 1 \\ k_{p} & k_{v}\end{array}\right]$ is a Hurwitz matrix, so that the error dynamics is exponentially stable in the classical linear sense. Indeed, with (8.259), it is easy to verify that along flows, the linear error dynamics corresponds to:

$$
\dot{e}=(\bar{A}+\overline{B K}) e .
$$

Unfortunately, such a selection, which disregards the possible negative effects of the
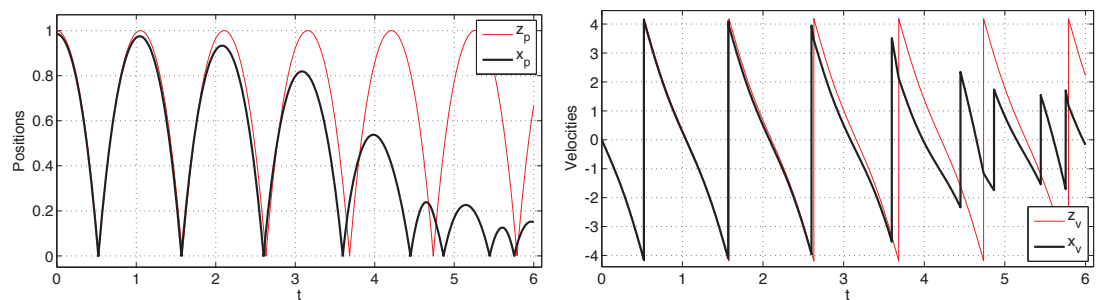

Figure 8.33: Simulation of the destabilizing effects of the linear tracking controller for the hammer example. Left plot: positions; Right plot: velocities. Reference hammer: red; controlled hammer: black.

jump dynamics, not only fails to guarantee convergence to zero of the error but it even induces instability of the zero equilibrium of the error dynamics. This is not formally proven here but a proof could be constructed following similar steps to those of the proof of the instability of the bouncing ball system analyzed in [FOR 11a, Example 1]. Here we show the instability phenomenon via the simulations of Figure 8.33, where the following values of the parameters have been used for simplicity: $k_{s}=1, k_{f}=1$ and $k_{p}=k_{v}=-1$. The values above lead to a simplified control law where the action of the spring and the dissipation of the viscous friction are directly exploited by the stabilizer:

$$
\begin{aligned}
u & =\alpha_{z}(z)-\alpha_{x}(x)+\bar{K}(x-z) \\
& =g \cos \left(x_{p}\right)-g \cos \left(z_{p}\right)-k_{s} z_{p}+k_{f} x_{v}+\left[\begin{array}{ll}
k_{p} & k_{v}
\end{array}\right]\left[\begin{array}{c}
x_{p}-z_{p} \\
x_{v}-z_{v}
\end{array}\right] \\
& =g \cos \left(x_{p}\right)-g \cos \left(z_{p}\right)-x_{p}-z_{v} .
\end{aligned}
$$


Figure 8.33 clearly illustrates the instability of the set where $e=0$. Indeed, despite the extremely small initial mismatch of the initial conditions $x(0,0)=(0.985,0)$, $z(0,0)=(1,0)$ (see left plot), the tracking performance is clearly lost by the linear closed loop. Similar transients are experienced for decreasing values of the initial tracking error.

\subsubsection{Using a mirrored reference to design a hybrid stabilizer}

The defective behavior induced on the hybrid tracking system (8.256)-(8.258) by the linear tracking controller (8.261) illustrated in the previous section can be avoided by ensuring that the error dynamics does not exhibit undesirable spikes arising from the non-negligible jumps in the velocities occurring when the impact times are not perfectly aligned. To this aim, we convert the linear error selection $e=x-z$ in (8.259) into a generalized error arising from the suggestive intuition that reference hammer is mirrored through the impacting surface (namely its sign is changed) thereby clearly obtaining a (temporary) inversion of the undesirable effects of the impact on the linear error dynamics (recall from (8.257), (8.258) that the impact effect is to change the sign of the corresponding variable). More specifically, we introduce the following generalized error:

$$
e:=x-q z
$$

where the logic variable $q$ is either 1 (no mirroring) or -1 (mirroring), that is $q \in$ $\{-1,1\}$, and at each impact of either $z$ or $x, q$ is toggled as follows:

$$
q^{+}=-q,
$$

while during flows it is kept constant:

$$
\dot{q}=0 .
$$

The hybrid control input is thus given by the following generalization of the feedback law (8.259), based on the generalized error (8.262), which can be simplified in similar ways to (8.261) as follows:

$$
\begin{aligned}
u & =\bar{K} e-\alpha_{x}(x)+q \alpha_{z}(z) \\
& =g \cos \left(x_{p}\right)-q g \cos \left(z_{p}\right)-q z_{v}-x_{p} .
\end{aligned}
$$

We emphasize that the hybrid control (8.265) differs from (8.261) only by the multiplicative factor $q$ on terms that depend on $z$. The following property motivates the introduction of the logic variable $q$ and of the generalized error in (8.262).

Property 1 Consider the hybrid closed-loop system (8.256)-(8.282), (8.263)-(8.265) and the definition of $e$ in (8.262). Then, for any value of the state $(x, z, q, \sigma)$ in the jump set,

$$
e^{+}=e \text { or } e^{+}=-e .
$$


Proof. The two relations can be verified by inspection. In particular, whenever $z$ impacts we have

$$
\begin{aligned}
e^{+} & =x-\left(q^{+}\right) z^{+}=x-(-q)(-z) \\
& =x-q z=e
\end{aligned}
$$

while at an impact of $x$ we have

$$
\begin{aligned}
e^{+} & =x^{+}-\left(q^{+}\right) z=-x-(-q) z \\
& =-(x-q z)=-e .
\end{aligned}
$$

Using Property 8.262 and the unifying Lyapunov result given in Section 8.6.1, we can provide the following theorem whose proof is given in Section 8.6.2.

Theorem 48 There exists $\gamma \geq 1$ and $\lambda>0$ such that every solution to the hybrid closed-loop system (8.256)-(8.258), (8.262)-(8.265) satisfies

$$
|e(t, j)| \leq \gamma \exp (-\lambda(t+j))|e(0,0)| .
$$

The reader will notice that the theorem is a consequence of the fact that the error norm does not increase at jumps, by Property 1, and that it decreases during flows, since the generalized error $e=x-q z$ still preserves the Hurwitz dynamics $\dot{e}=(\bar{A}+\overline{B K}) e$, together with the persistence of flow induced by the average dwell-time logic.

In general, the convergence of the generalized error to 0 is not sufficient to claim tracking, which is recovered, at least during flows, by combining Theorem 48 with the following proposition. The proposition establishes that the system cannot flow and satisfy concurrently $e=0$ and $x=-z$, which recovers tracking, i.e. $x=z$, whenever $e=0$ and the system flows. We emphasize that no conditions are imposed on $z$ and $x$ at jumps, where the relaxation of the identity $x=z$ is a necessary condition to allow for an instantaneous mismatch of the velocities in-between the two jumps of $x$ and $z$ (even though they occur at the same ordinary time).

Proposition 16 Consider any solution to the hybrid closed-loop system (8.256)(8.258), (8.262)-(8.265) starting from $e(0,0)=0$. Then, $e(t, j)=0$ for all $(t, j)$ in $\operatorname{dom}(e)$ and the solution cannot flow unless $x(t, j)=z(t, j)$. 
Proof. By Theorem 48, the set where $e=0$ is strongly forward invariant for the system dynamics. Thus, we only need to prove that $x=z$ if $e=0$ and the system flows. To this aim, first note that $e=x-q z=0$ implies that either $x=z$ or $x=-z$. In the former case the result is proven; in the latter case we necessarily have $x=-z=(0,0)=z$, as shown next. Since the sets $C_{x}$, $C_{z}$ where flow of $x$ and $z$ is allowed only contain non-negative positions, then $x_{p}=-z_{p}$ implies $x_{p}=z_{p}=0$. Moreover, since $x_{v}=-z_{v}$ and and none of the two hammers can flow outside of the flow set, we also get $x_{v}=z_{v}=0$, which implies $x=-z=(0,0)$.
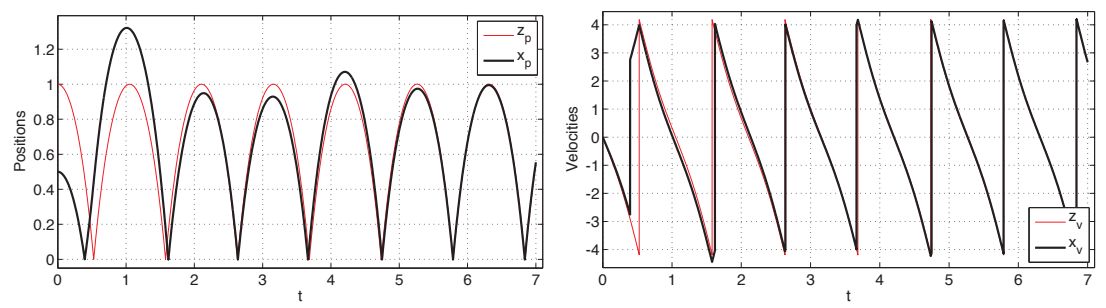

Figure 8.34: Simulation of the hybrid tracking controller for the hammer example. Left plot: positions; Right plot: velocities. Reference hammer: red; controlled hammer: black.

Figure 8.34 shows a simulation with the same parameters as those used in the defective case of Figure 8.33 but with the hybrid stabilizer (8.265). The red trace represents the periodic motion of the reference hammer $z$, while the black trace shows the motion of the controlled hammer. Both systems start with zero speed and with initial positions $z_{p}(0,0)=2 x_{p}(0,0)=1$ which leads to a significant initial mismatch (see left plot). Exponential convergence to zero of the error can be clearly appreciated from the simulation results.

\subsection{Global tracking of a Newton's cradle}

In our second example we move on to a mechanical system with two degrees of freedom. The motivation and model used here is strongly inspired by the work in [MEN 02]. From the point of view of the mirrored reference, the more complicated impact law of this example requires a different definition, which is perhaps less explicit but is a useful first step toward the more general description of the next two sections. 


\subsubsection{The reference cradle}

Let us consider the reference dynamics $\mathcal{Z}$ given by a simplified Newton's cradle adopting the model used in [MEN 02], whose two equal pendulums have unit mass, unit length, and are subject to the gravity acceleration $g$ (see Figure 8.35). Using $z_{1}$ and $z_{2}$ to denote the angular deviation of the two pendulums from the vertical position (as customary, a positive $z_{i}$ corresponds to a counter-clockwise rotation), the continuous dynamics of each pendulum is described by

$$
\ddot{z}_{i}=-g \sin \left(z_{i}\right), \quad i \in\{1,2\}
$$

which comes from recognizing that the torque exerted on the pendulum hinge arises

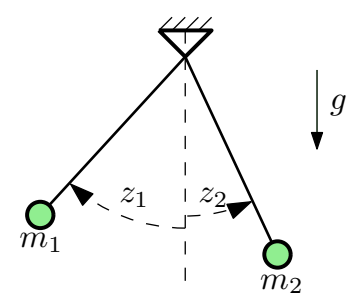

Figure 8.35: A simple model of the Newton's cradle.

from the projection of the gravity force on the orthogonal plane to the hinge constraint. To simplify our formulation, we assume that the swinging of the two cradles is limited to the left and right side of Figure 8.270 for the first and second masses $z_{1}, z_{2}$, which can be obtained by restricting the hybrid motion to the following closed set:

$$
\mathcal{K}=\left\{z \in \Re^{4}: z_{1} \geq-\pi, z_{2} \leq \pi\right\},
$$

and simply terminating solutions outside this set by not allowing them to flow or jump outside $\mathcal{K}$ (this parallels the restriction of the hammer positions to the set $[0, \pi]$ enforced in the example of the previous section). A convenient reformulation of dynamics (8.270) restricted to $\mathcal{K}$ is given by

$$
\dot{z}=\bar{A} z+\bar{B} \alpha(z) \quad z \in C_{z}:=\left\{z \in \Re^{4}: z_{1} \leq z_{2}\right\} \cap \mathcal{K},
$$

where the state vector $z=\left[\begin{array}{c}z_{p} \\ z_{y}\end{array}\right]$ has components $z_{p}:=\left[\begin{array}{l}z_{1} \\ z_{2}\end{array}\right]$ (positions of the two pendulums) and $z_{v}:=\left[\begin{array}{c}z_{3} \\ z_{4}\end{array}\right]:=\left[\begin{array}{c}z_{1} \\ \dot{z}_{2}\end{array}\right]$ (corresponding velocities). Moreover the matrices in (8.271) represent the (decoupled) double integrator dynamics of each pendulum. This dynamics is conveniently represented by using the Kronecker product as

$$
\bar{A}:=\left[\begin{array}{ll}
0 & 1 \\
0 & 0
\end{array}\right] \otimes I_{2}, \quad \bar{B}:=\left[\begin{array}{l}
0 \\
1
\end{array}\right] \otimes I_{2}, \quad \alpha(z):=-g\left[\begin{array}{c}
\sin \left(z_{1}\right) \\
\sin \left(z_{2}\right)
\end{array}\right] .
$$


An impact between the two spheres occurs when the positions satisfy $z_{1}=z_{2}$ and the velocities satisfy $\dot{z}_{1} \geq \dot{z_{2}}$ so that intuitively the two masses are at the same location with colliding velocities. The effect of the impact is to swap the velocities of the two spheres so that colliding spheres will move away from each other after the impact. This phenomenon is modeled by

$$
z^{+}=\left[\begin{array}{c}
z_{p} \\
M z_{v}
\end{array}\right]=\bar{M} z \quad z \in D_{z}:=\left\{z \in \Re^{4}: z_{1}=z_{2}, z_{3} \geq z_{4}\right\} \cap \mathcal{K} .
$$

where $M:=\left[\begin{array}{ll}0 & 1 \\ 1 & 0\end{array}\right]$, and $\bar{M}:=\left(I_{2} \otimes M\right)=\left[\begin{array}{cc}M & 0 \\ 0 & M\end{array}\right]$. Note that at impacts, namely whenever $z \in D_{z}$, we have $M z_{p}=z_{p}$, indeed by assumption $z_{1}=z_{2}$ in $D_{z}$ and swapping these two quantities causes no effect.

Note that in (8.273) we intentionally characterize the jump set $D_{z}$ as a closed set so that suitable regularity properties of the solution set of our hybrid system are satisfied. Due to this reason, when $\left(z_{1}, z_{3}\right)=\left(z_{2}, z_{4}\right)$ the system may exhibit Zeno solutions (this is when the two cradles are perfectly synchronized). To avoid this side effect of the adopted modeling framework, we regularize the space of solutions by introducing the dwell-time logic (8.253) in the closed loop. Note that neither $N$ nor $\rho$ will be used in the proposed control design and these quantities may be arbitrarily large.

\subsubsection{The controlled cradle}

The controlled Newton's cradle $\mathcal{X}$ shares the dynamics of the reference cradle but its flow can be governed by using a force input $u$. Using the state vector $x \in \Re^{4}$ and the input vector $u:=\left[\begin{array}{ll}u_{1} & u_{2}\end{array}\right]^{T} \in \Re^{2}$, the controlled cradle dynamics is given by

$$
\begin{aligned}
& \dot{x}=\bar{A} x+\bar{B}(\alpha(x)+u), \quad x \in C_{x}:=\left\{x \in \Re^{4}: x_{1} \leq x_{2}\right\} \cap \mathcal{K} \\
& x^{+}=\bar{M} x, \quad x \in D_{x}:=\left\{x \in \Re^{4}: x_{1}=x_{2}, x_{3} \geq x_{4}\right\} \cap \mathcal{K} .
\end{aligned}
$$

We consider an initial design for the control input $u$ provided by a linear feedback from a suitable error function plus cancellation of the nonlinear term $\alpha$, corresponding to

$$
\begin{aligned}
e & =x-z \\
u & =\bar{K} e-\alpha(x)+\alpha(z) .
\end{aligned}
$$

The gain $\bar{K}$ is selected in such a way that the linear flow dynamics is governed by a Hurwitz transition matrix $\bar{A}+\overline{B K}$. In particular, to preserve the peculiar structure of the reference/controlled flow dynamics (a parallel interconnection of double integrators), the gain $\bar{K}$ is selected of the following form (compare to (8.272)):

$$
\bar{K}:=K \otimes I_{2}=\left[k_{1} k_{2}\right] \otimes I_{2},
$$

where $k_{1}, k_{2}$ guarantee that $A_{c l}=\left[\begin{array}{cc}0 & 1 \\ k_{1} & k_{2}\end{array}\right]$ is a Hurwitz matrix (namely they are both strictly negative or, in other words, $K$ is any stabilizing gain for the double integrator). As a result, we get $\bar{A}+\overline{B K}=\left[\begin{array}{cc}0 & 1 \\ k_{1} & k_{2}\end{array}\right] \otimes I_{2}$, whose peculiar structure is exploited next in the hybrid modification of the input $u$. 


\subsubsection{Using a mirrored reference to design a hybrid stabilizer}

Taking inspiration from the approach adopted in Section 8.2.5, we introduce a mirrored reference depending on a flag variable $q$ which is toggled at each jump as follows:

$$
\left\{\begin{array}{l}
\dot{q}=0 \\
q^{+}=1-q .
\end{array}\right.
$$

In particular, we define the mirrored reference $m(q, z)$ for all $z, q \in \Re^{4} \times\{0,1\}$ as

$$
m(q, z):= \begin{cases}z & \text { if } q=0 \\ \bar{M} z & \text { if } q=1\end{cases}
$$

and we emphasize that $m(0, z)=z$ while $m(1, z)$ is more involved: whenever $z \in$ $D_{z}, m(1, q)$ corresponds to the inversion of the jump map in (8.273) but when $z \notin D_{z}$, the map introduces a peculiar swap of both the positions and the velocities of the two cradles.

Paralleling the hammer on the wall solution, the mirrored reference allows to define the error function in such a way that it satisfies a generalization of the conditions established in Property 1. In particular, we exchange the linear feedback in (8.275) for the following one (note that the two definitions coincide when $q=0$ ):

$$
\begin{aligned}
e & =x-m(q, z) \\
u & =\bar{K} e-\alpha(x)+\bar{B}^{T} m(q, \bar{B} \alpha(z)) .
\end{aligned}
$$

Summarizing, we can lump into a single hybrid dynamical system the dynamic equations (8.271), (8.273), (8.274), the dwell-time logic (8.253) and the dynamics of the automaton $q$ in (8.277) to get the overall closed-loop system having flow dynamics given by (8.279) and

$$
\left\{\begin{aligned}
\dot{z} & =\bar{A} z+\bar{B} \alpha(z) \\
\dot{x} & =\bar{A} x+\bar{B}(\alpha(x)+u) \quad(z, x, q, \sigma) \in C_{z} \times C_{x} \times\{0,1\} \times[0, N] . \\
\dot{q} & =0 \\
\dot{\sigma} & \in[0, \rho]
\end{aligned}\right.
$$

Moreover an impact of the reference cradle $\mathcal{Z}$ is given by

$$
\left\{\begin{array}{l}
z^{+}=\bar{M} z \\
x^{+}=x \\
q^{+}=1-q \\
\sigma^{+}=\sigma-1
\end{array} \quad(z, x, q, \sigma) \in D_{z} \times\left(C_{x} \cup D_{x}\right) \times\{0,1\} \times[1, N],\right.
$$

while impacts of the controlled cradle $\mathcal{X}$ are described by

$$
\left\{\begin{array}{l}
z^{+}=z \\
x^{+}=\bar{M} x \\
q^{+}=1-q \\
\sigma^{+}=\sigma-1
\end{array} \quad(z, x, q, \sigma) \in\left(C_{z} \cup D_{z}\right) \times D_{x} \times\{0,1\} \times[1, N] .\right.
$$


Note that a synchronous impact of the reference and controlled cradles is characterized by two consecutive jumps of the hybrid closed loop, given by either (8.281) followed by (8.282), or vice versa.

The following property motivates the introduction of the mirrored reference variable in (8.279) and allows to prove the tracking proposition given below.

Property 2 For any matrix $P \in \Re^{2 \times 2}$, define $\bar{P}=P \otimes I_{2}$. Consider the hybrid closed loop (8.280)-(8.282) and the definition of e in (8.279). Then the jump map ensures that for all e in the jump set

$$
\left(e^{+}\right)^{T} \bar{P} e^{+}=e^{T} \bar{P} e .
$$

Proof. First note that from (8.278) we have $m(q, z)=\left(I_{2} \otimes \tilde{M}(q)\right) z$, where $\tilde{M}(0)=I$ and $\tilde{M}(1)=M$. Moreover, using $(A \otimes B)(C \otimes D)=(A C) \otimes(B D)$, we have

$$
\begin{aligned}
\overline{M P M} & =\left(I_{2} \otimes \tilde{M}(q)\right)\left(P \otimes I_{2}\right)\left(I_{2} \otimes \tilde{M}(q)\right) \\
& =\left(I_{2} \otimes \tilde{M}(q)\right)(P \otimes \tilde{M}(q))=P \otimes \tilde{M}(q)^{2} \\
& =P \otimes I_{2}=\bar{P}
\end{aligned}
$$

and, by direct calculation, we can verify that $\overline{M M}=I_{4}$.

Consider now the case when $z$ jumps. Then, since also $q$ toggles, it is easily verified that $e^{+}=x-m\left(q^{+}, z^{+}\right)=x-m\left(q, \bar{M} z^{+}\right)=x-m(q, \overline{M M} z)=e$ and (8.283) follows straightforwardly. When $x$ jumps, instead, we have $e^{+}=$ $x^{+}-m\left(q^{+}, z\right)=\bar{M}(x-m(q, z))=\bar{M} e$ and (8.283) follows from identity (8.284).

Using Property 2 and the unifying Lyapunov result given in Section 8.6.1, we are able to state the following result which establishes global exponential stability of the set where the hybrid error $e$ is zero for the hybrid closed-loop system. Its proof is given in Section 8.6.2.

Theorem 49 There exists $\gamma \geq 1$ and $\lambda>0$ such that every hybrid solution $\xi$ to the closed-loop system (8.279)-(8.282) satisfies

$$
|e(t, j)| \leq \gamma \exp (-\lambda(t+j))|e(0,0)| .
$$


In general the convergence of the generalized error to 0 does not necessarily guarantee tracking. This is a consequence of the fact that for some configurations of reference and controlled systems $x \neq z$ despite $e=0$. Tracking is recovered indeed through the combination of the exponential decrease of the error in (8.285) and the following statement which establishes that $e=0$ implies $x=z$ away from state configurations related to impacts (i.e. for almost all time).

Proposition 17 Consider any solution $\xi$ to the hybrid closed-loop system (8.279)(8.282) starting from an initial condition satisfying $e(0,0)=0$. Then $e(t, j)=0$ for all $(t, j) \in \operatorname{dom}(e)$ and the solution cannot flow unless $x(t, j)=z(t, j)$.

Proposition 17 establishes that in the attractor, where $e=0$, all solutions that flow are characterized by $x-z=0$. This establishes tracking during flows and allows for a (necessary) mismatch of $x$ and $z$ at jumps. Note however that at jumps (namely in $D_{z}$ and $D_{x}$ ) one has $z_{p}=x_{p}$ so that tracking of the positions is guaranteed at all (hybrid) times, while tracking of the velocities is only guaranteed at times that are not impact times.

Proof. The fact that $e$ remains zero for all (hybrid) times comes from forward invariance of the set where $e=0$, which is a consequence of stability. Regarding the second statement of the proposition, assume that it does not hold, namely consider the case $e(t, j)=0$ and $q(t, j)=1$ for some $(t, j)$ and let us omit $(t, j)$ for simplicity. Then we have $x=\bar{M} z$, which implies $x_{1}=z_{2}, x_{2}=z_{1}$. This last relation, combined with the flow set in (8.271) and (8.274) implies that it is only possible to flow if $x_{1}=x_{2}=z_{1}=z_{2}$, namely all pendulums are at the same position and the system is on the jump set.

Moreover, $x=\bar{M} z$ implies a similar relation among the velocities: $x_{3}=z_{4}$, $x_{4}=z_{3}$. This second relation together with the flow dynamics implies that the system can flow only if $x_{3}=x_{4}=z_{3}=z_{4}$. Summarizing, if $q=1$ then the system can only flow when $x=z$. If $q=0$, then $x=z$ because $e=0$.

\subsubsection{Simulations}

To illustrate the effectiveness of the proposed approach we simulate the hybrid closed-loop system (8.279)-(8.282) from the initial conditions $z_{0}=\left[\begin{array}{llll}-0.2 & 1 & 0 & -1\end{array}\right]^{T}$

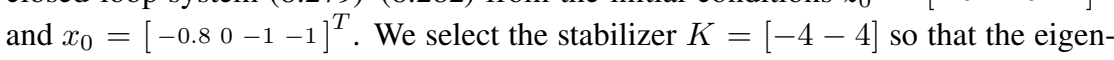
values of $A_{c l}$ are both placed in -2 and this corresponds to the expected rate of convergence of the error dynamics along flows. 

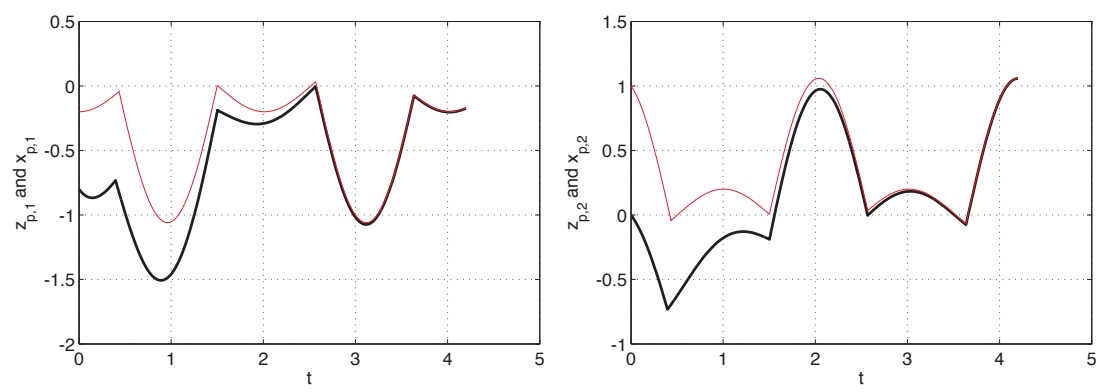

Figure 8.36: Positions (left) and velocities (right) of the Newton cradles for $q(0,0)=$ 0
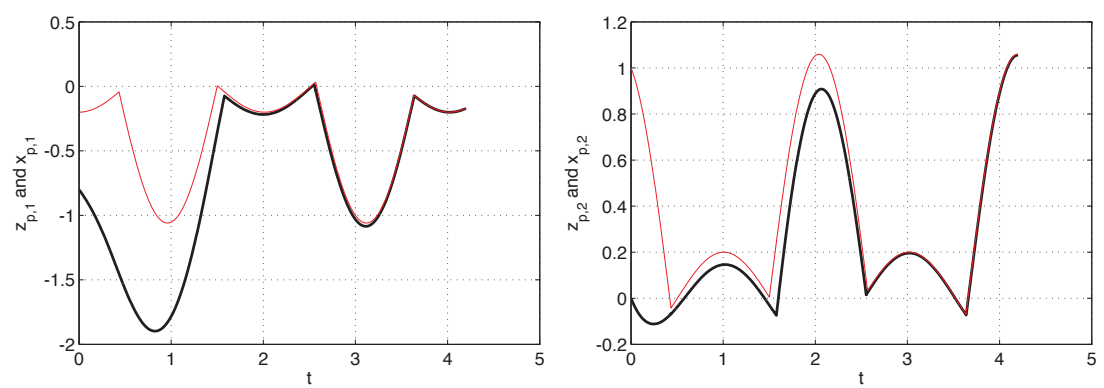

Figure 8.37: Positions (left) and velocities (right) of the Newton cradles for $q(0,0)=$ 1.

Figures 8.36 shows the response starting from $q(0,0)=0$, projected in the $t$ direction of the hybrid time domain. Since $q(0,0)=0$, the feedback stabilizer initially focuses on the real target. Mirroring allows to suitably treat the mismatch of the ordinary times when the impacts of $z$ (red thin trace) and $x$ (black bold trace) occur. For example, the first impact of the $x$ cradle (black curve) occurs at $\left(t_{1}, 0\right) \approx(0.395,0)$ while the first impact of the $z$ cradle (red curve) occurs at $\left(t_{2}, 1\right) \approx(0.435,1)$. During the flow between these two impacts, the control law tracks the mirrored target, namely $q(t, 1)=1$ for all $t \in\left[t_{1}, t_{2}\right]$. Figure 8.37 shows a second simulation starting from $q(0,0)=1$. Clearly, the response of the $z$ cradle (red curves) remains unchanged. Since $q(0,0)=1$, the initial target tracked by the controller is the mirrored one. This fact is evident in the both plots of Figure 8.37 which reveal that the tracker starts flowing in the opposite direction as compared to the previous case and after the first impact of the $z$ cradle (red curve), occurring at ordinary time $\left(t_{1}, 0\right)=(0.435,0), q$ becomes zero and a similar transient to that of the previous case is experienced. Note 
also the mismatch between the two subsequent impacts around ordinary time $t \approx 1.5$. Also in this case the mirroring action allows to maintain the regularity of the transient response despite the impact mismatch.

\subsection{Global tracking in planar triangles}

One simplifying feature of the tracking algorithms of Sections 8.2 and 8.3 is that impacts always occur at a specific surface in the position subspace of the state space so that one can rely on a suitable mirror of the target with respect to that surface and recover the linear behavior of the error dynamics. This idea can be generalized to more complicated settings where impacts may occur on different surfaces, as long as each surface is associated to the correct mirrored image and suitable compositions of mirrors are performed when consecutive impacts on different surfaces are experienced. The above intuitive behavior has been addressed in [FOR 11c] for several planar regions (or "billiards") having special shapes. Moreover, for the case of rectangular billiards, a comprehensive treatment is given in [FOR 11a]. In this section we revisit the results given in [FOR 11c, Section V.G] about dynamics restricted to subsets of the plane corresponding to equilateral triangles, and we show that global asymptotic tracking can be proven using the unified Lyapunov techniques given in Section 8.6.

\subsubsection{The reference mass}

We consider a tracking problem for a reference mass $z$ moving in a planar region delimited by an equilateral triangle. The reference mass $z=\left(z_{p}, z_{v}\right) \in \Re^{4}$ is such that its position sub-vector $z_{p}$ is confined to the region $\mathcal{F} \subset \Re^{2}$ defined by

$$
\mathcal{F}=\left\{s \in \Re^{2}: \forall i \in \mathcal{I},\left\langle F_{i}, s-s_{\circ}\right\rangle \leq 1\right\},
$$

with $F_{i} \in \Re^{2}, i \in\{1,2,3\}$ denoting the vectors characterizing each wall of the triangle $\mathcal{F}$ and $s_{\circ} \in \Re^{2}$ being a fixed point in the interior of the region characterizing the position of the triangle in the plane (see Figure 8.38).

Following the approach in the previous section, we consider a quasi-linear reference dynamics given by

$$
\dot{z}=\bar{A} z+\bar{B} \alpha\left(z_{p}\right) \quad z \in C_{z}:=\mathcal{K} \subset \mathcal{F} \times \Re^{2}
$$

where $\bar{A}:=\left[\begin{array}{ll}0 & 1 \\ 0 & 0\end{array}\right] \otimes I_{2}, \bar{B}:=\left[\begin{array}{l}0 \\ 1\end{array}\right] \otimes I_{2}, I_{2}$ is the $2 \times 2$ identity matrix and $\alpha\left(z_{p}\right) \in$ $\Re^{2}$ is a known nonlinear term that depends only on the position sub-vector $z_{p}$. The restriction of the flow and jump dynamics to the closed set $\mathcal{K}$ keeps the motion of the reference away from the "corners" of the triangular billiard, i.e. the points $s_{p}$ such 


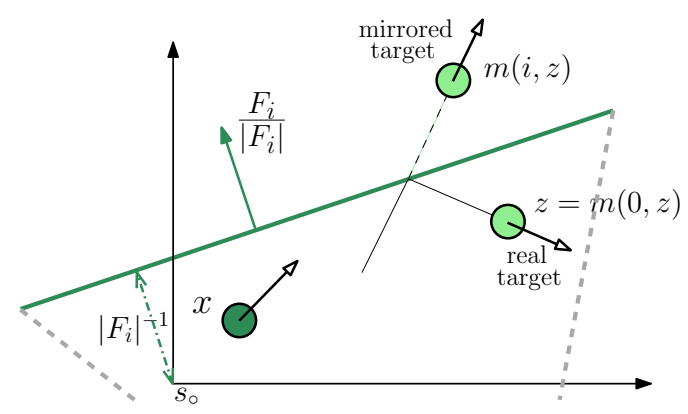

Figure 8.38: The triangular region $\mathcal{F}$ and the mirror of the target $z$ with respect to one wall.

that $\left\langle F_{i}, s_{p}-s_{\circ}\right\rangle=\left\langle F_{j}, s_{p}-s_{\circ}\right\rangle=1$ for $i, j \in \mathcal{I}, i \neq j$. This assumption rules out places where the impact dynamics may be a set valued map.

To ensure that the motion of the reference dynamics $z=\left(z_{p}, z_{v}\right)$ is restricted to the region $\mathcal{F} \times \Re^{2}$, an impact occurs when the position $z_{p}$ belongs to the boundary $\partial \mathcal{F}$ of the triangle $\mathcal{F}$ and the velocity $z_{v}$ satisfies $\left\langle F_{i}, z_{v}\right\rangle \geq 0$, for some $i \in\{1,2,3\}$, namely the mass bounces against the $i$-th wall represented by $F_{i}$. The impact on the $i$ th wall determines the inversion of the component of $z_{v}$ parallel to $F_{i}$ (namely the normal component to the wall). In [FOR 11b, FOR 11c, FOR 11a], such an impact was modeled by suitably combining two rotation matrices and the reflection matrix $\left[\begin{array}{cc}1 & 0 \\ 0 & -1\end{array}\right]$. Here, based on the derivations in [STR 03, page 220], we use the following simpler (and equivalent) formulation:

$$
M(i):=I_{2}-2 \frac{F_{i} F_{i}^{T}}{\left|F_{i}\right|^{2}},
$$

where $I_{2}$ is the identity matrix and $\left|F_{i}\right|=\sqrt{F_{i}^{T} F_{i}}$. Based on [STR 03, page 220], is is easily verified that $M(i)$ inverts the component of $z_{v}$ parallel to $F_{i}$. Thus, using the definitions

$$
\begin{aligned}
c(i) & :=F_{i} \cdot 2\left(1+F_{i}^{T} s_{\circ}\right) /\left|F_{i}\right|^{2} \\
\bar{M}(i) & :=I_{2} \otimes M(i) \\
\bar{c}(i) & :=\left[\begin{array}{ll}
c(i)^{T} & 0_{1 \times 2}
\end{array}\right]^{T}=\left[\begin{array}{l}
1 \\
0
\end{array}\right] \otimes c(i) \\
m(i, z) & :=\bar{M}(i) z+\bar{c}(i)
\end{aligned}
$$

the impact dynamics can be written compactly as

$$
z^{+}=m(i, z) \quad z \in D_{z}^{(i)}
$$


where for each $i \in\{1,2,3\}$

$$
\begin{aligned}
D_{z}^{(i)} & :=\left\{z \in \mathcal{K}:\left\langle F_{i}, z_{p}-s_{\circ}\right\rangle=1,\left\langle F_{i}, z_{v}\right\rangle \geq 0\right\} \\
D_{z} & :=\bigcup_{i \in\{1,2,3\}} D_{z}^{(i)}=\partial \mathcal{F} \times \Re^{2} \cap \mathcal{K} .
\end{aligned}
$$

Finally, as in the previous sections, we augment the system dynamics with the average dwell-time logic (8.253) having state $\sigma$ and introduced in Section 8.2.2, to rule out Zeno solutions that occur when the reference mass impacts a wall $F_{i}$ with zero orthogonal component of $z_{v}$ to $F_{i}$ (namely it slides along the wall). Similar to before, the parameters of the dwell time logic do not appear in the tracking controller construction but are essential for constructing the Lyapunov function establishing exponential convergence to zero of the error dynamics.

\subsubsection{The controlled mass}

Similar to the controlled Newton cradle of Section 8.3.2, the controlled mass shares the same dynamics as the reference mass but a force input $u$ is available to suitably steer it towards the reference one during flows:

$$
\begin{array}{ll}
\dot{x}=\bar{A} x+\bar{B}(\alpha(x)+u), & x \in C_{x}:=\mathcal{F} \times \Re^{2} \\
x^{+}=m(i, x) & x \in D_{x}^{(i)}, \quad i=\{1,2,3\},
\end{array}
$$

where for each $i \in\{1,2,3\}$,

$$
\begin{aligned}
D_{x}^{(i)} & :=\left\{x \in \mathcal{F} \times \Re^{2}:\left\langle F_{i}, x_{p}-s_{\circ}\right\rangle=1,\left\langle F_{i}, x_{v}\right\rangle \geq 0\right\} \\
D_{x} & :=\bigcup_{i \in\{1,2,3\}} D_{x}^{(i)}=\partial \mathcal{F} \times \Re^{2} .
\end{aligned}
$$

Before introducing the mirrored references and the hybrid tracking law, it is convenient to proceed similarly to the Newton cradle by introducing an initial design for the control input $u$ consisting in a linear error feedback plus cancellation of the nonlinear term $\alpha$ :

$$
\begin{aligned}
e & =x-z \\
u & =\bar{K} e-\alpha(x)+\alpha(z) .
\end{aligned}
$$

Once again the gain is selected as

$$
\bar{K}:=K \otimes I_{2}=\left[k_{1} k_{2}\right] \otimes I_{2},
$$

where $k_{1}, k_{2}$ guarantee that $A_{c l}=\left[\begin{array}{cc}0 & 1 \\ k_{1} & k_{2}\end{array}\right]$ is a Hurwitz matrix. With this selection we have $\bar{A}+\overline{B K}=\left[\begin{array}{cc}0 & 1 \\ k_{1} & k_{2}\end{array}\right] \otimes I_{2}$, whose peculiar structure is exploited next in the hybrid modification of the input $u$. 


\subsubsection{Using a family of mirrored references to design a hybrid stabilizer}

Differently from the solutions in Sections 8.2.5 and 8.3.3 the triangle case cannot be solved by introducing a single mirror of the reference mass $z$. Indeed, since the triangular billiard has three walls, at least three mirrored images are necessary to generalize the hybrid tracking techniques of the previous sections. It turns out that 12 mirrored images will be necessary to solve our problem due to compositions of mirroring, as clarified below. Since we will introduce many mirrors in the sequel, the state variable $q$ is not anymore a flag but it becomes a logic variable taking values in

$$
\mathcal{Q}=\{0,1,2,3,21,31,12,32,13,23,121,131,232\},
$$

characterizing the real target $(q=0)$ and twelve suitable mirrors (see also Figures 8.38 and 8.39). This generalization has also been used in [FOR 11c, Section V.G] and an algebraic proof of the effectiveness of the scheme has been given. Here we rewrite the proof using graphical arguments which perhaps simplify the understanding of the rationale behind the hybrid scheme.

According to the scheme of Figure 8.39, for each $z \in \mathcal{K}$ (a possible one being suitably represented in the figure and comprising position and velocity), we define the mirrored references as $m(0, z)=z, m(q, z)$ as specified in (8.289d) for $q \in\{1,2,3\}$ and the remaining mirrors as the following compositions of the three basic mirrors (refer to Figure 8.39 for an intuitive understanding of each mirror):

$$
\begin{array}{ll}
m(21, z)=m(2, m(1, z)), & m(31, z)=m(3, m(1, z)), \\
m(12, z)=m(1, m(2, z)), & m(32, z)=m(3, m(2, z)), \\
m(13, z)=m(1, m(3, z)), & m(23, z)=m(2, m(3, z)), \\
m(121, z)=m(1, m(2, m(1, z))), & m(131, z)=m(1, m(3, m(1, z))), \\
m(232, z)=m(2, m(3, m(2, z))) . &
\end{array}
$$

Proceeding in ways similar to Section 8.3 .3 we can then introduce the generalized error variable and the hybrid tracking law which coincides with equation (8.279) and is reproduced here for ease of presentation:

$$
\begin{aligned}
e & =x-m(q, z) \\
u & =\bar{K} e-\alpha(x)+\bar{B}^{T} m(q, \bar{B} \alpha(z)) .
\end{aligned}
$$

While in the previous two sections the variable $q$ was a flag that was easily toggled between 0 and 1 at each impact, for the triangular billiard case we need to suitably updating the logic variable $q$ in such a way that a parallel result to Property 2 holds across jumps. To this aim, following the derivations in [FOR 11c, FOR 11a], we introduce two automata $\delta_{z}: \mathcal{Q} \times\{1,2,3\} \rightarrow \mathcal{Q}$ and $\delta_{x}: \mathcal{Q} \times\{1,2,3\} \rightarrow \mathcal{Q}$ which indicate at each jump of $z$ or $x$, respectively, ${ }^{10}$ what should be the new value $q^{+}$of the logic

10. Note that in [FOR 11a] the two automata are actually the same due to the special planar regions considered there. 


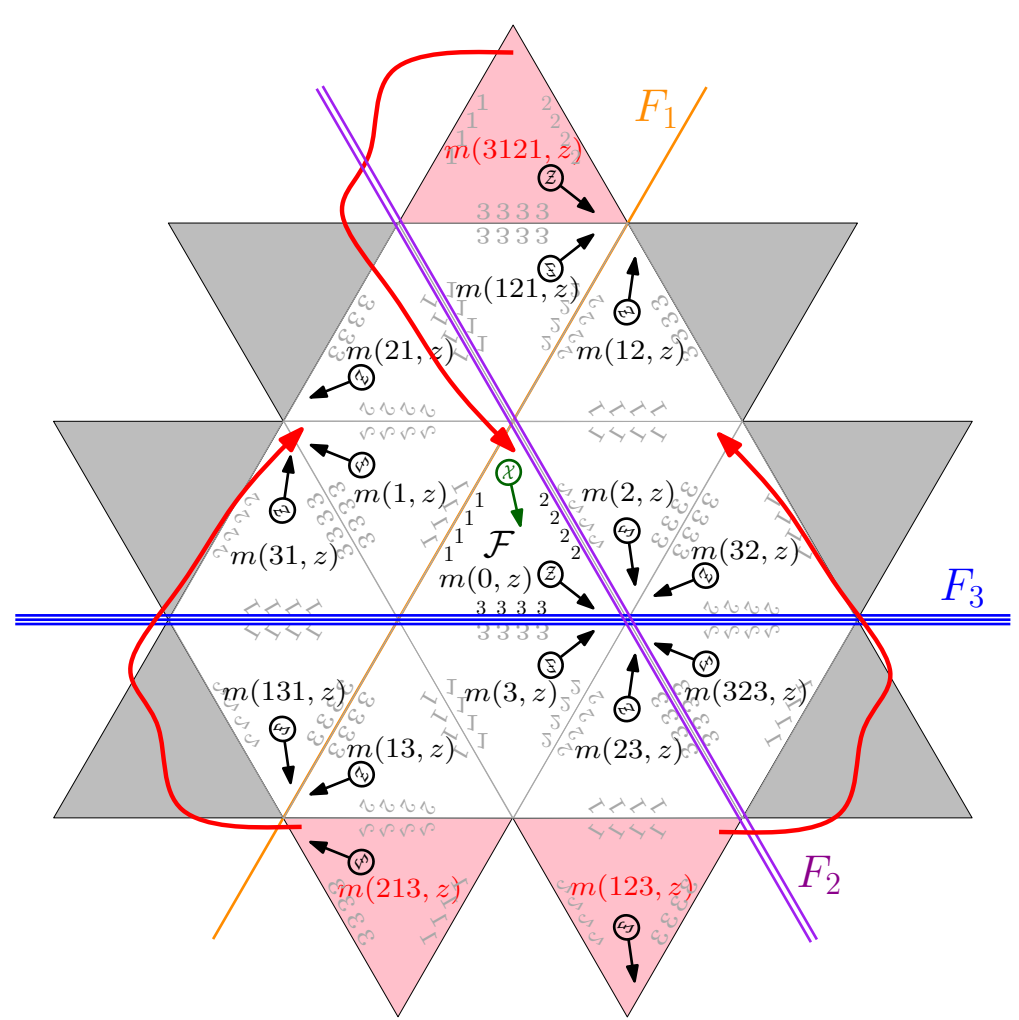

Figure 8.39: The triangular region of Section 8.4 and the twelve mirrored references.

variable $q$. Determining suitable automata is the most difficult aspect of the construction of this section and will be discussed later in conjunction with Property 3.

Summarizing, we can lump into a single hybrid dynamical system the dynamic equations (8.287), (8.290), (8.291), the controller (8.295), the dwell-time logic (8.253) and the dynamics of the automaton $q$ to get the overall hybrid closed-loop system having flow dynamics given by (8.295) and

$$
\left\{\begin{aligned}
\dot{z} & =\bar{A} z+\bar{B} \alpha(z) \\
\dot{x} & =\bar{A} x+\bar{B}(\alpha(x)+u) \quad(z, x, q, \sigma) \in C_{z} \times C_{x} \times \mathcal{Q} \times[0, N] \\
\dot{q} & =0 \\
\dot{\sigma} & \in[0, \rho]
\end{aligned}\right.
$$


Moreover an impact of the reference mass $\mathcal{Z}$ on the $i$-th wall is given by

$$
\left\{\begin{array}{l}
z^{+}=m(i, z) \\
x^{+}=x \\
q^{+}=\delta_{z}(q, i) \\
\sigma^{+}=\sigma-1
\end{array} \quad(z, x, q, \sigma) \in D_{z}^{(i)} \times\left(C_{x} \cup D_{x}\right) \times \mathcal{Q} \times[1, N],\right.
$$

while an impact of the controlled mass $\mathcal{X}$ on the $i$-th wall is described by

$$
\left\{\begin{array}{l}
z^{+}=z \\
x^{+}=m(i, x) \\
q^{+}=\delta_{x}(q, i) \\
\sigma^{+}=\sigma-1
\end{array} \quad(z, x, q, \sigma) \in\left(C_{z} \cup D_{z}\right) \times D_{x}^{(i)} \times \mathcal{Q} \times[1, N] .\right.
$$

Note that a synchronous impact of the reference and controlled masses is characterized by two consecutive jumps of the hybrid closed loop, given by either (8.297) followed by (8.298), or vice versa. Moreover, a simultaneous impact of the controlled mass on two walls (at a corner) may occur and is also characterized by two consecutive jumps of the hybrid closed loop, while a simultaneous impact of the reference mass on two walls (at a corner) is not possible because its motion is restricted to the closed set $\mathcal{K}$ which does not contain any corner.

The design of the hybrid tracking controller is completed by the selection of the automata $\delta_{z}, \delta_{x}$, which is carried out to ensure that whenever the reference mass $z$ or the controlled mass $x$ experience an impact, the quantity $e^{T} \bar{P} e$ involving the generalized error $e$ in (8.295) does not increase, as long as $\bar{P}$ has a suitable structure (this parallels, e.g., the property in (8.283) for the Newton cradle). In particular, the automata $\delta_{z}, \delta_{x}$ are determined graphically, based on the mirrored regions represented in Figure 8.39, and corresponds to the following lookup tables:

\begin{tabular}{|c|c|c|c|c|c|c|c|c|c|c|c|c|c|}
\hline$q$ & 0 & 1 & 2 & 3 & 21 & 31 & 12 & 32 & 13 & 23 & 121 & 131 & 232 \\
\hline \hline$\delta_{z}(q, 1)$ & 1 & 0 & 12 & 13 & 121 & 131 & 2 & $\mathbf{3}$ & 3 & $\mathbf{2}$ & 21 & 31 & $\mathbf{0}$ \\
\hline$\delta_{z}(q, 2)$ & 2 & 21 & 0 & 23 & 1 & $\mathbf{3}$ & 121 & 232 & $\mathbf{1}$ & 3 & 12 & $\mathbf{0}$ & 32 \\
\hline$\delta_{z}(q, 3)$ & 3 & 31 & 32 & 0 & $\mathbf{2}$ & 1 & $\mathbf{1}$ & 2 & 131 & 232 & $\mathbf{0}$ & 13 & 23 \\
\hline
\end{tabular}

\begin{tabular}{|c|c|c|c|c|c|c|c|c|c|c|c|c|c|}
\hline$q$ & 0 & 1 & 2 & 3 & 21 & 31 & 12 & 32 & 13 & 23 & 121 & 131 & 232 \\
\hline \hline$\delta_{x}(q, 1)$ & 1 & 0 & 21 & 31 & 2 & 3 & 121 & $\mathbf{2}$ & 131 & $\mathbf{3}$ & 12 & 13 & $\mathbf{0}$ \\
\hline$\delta_{x}(q, 2)$ & 2 & 12 & 0 & 32 & 121 & $\mathbf{1}$ & 1 & 3 & $\mathbf{3}$ & 232 & 21 & $\mathbf{0}$ & 23 \\
\hline$\delta_{x}(q, 3)$ & 3 & 13 & 23 & 0 & $\mathbf{1}$ & 131 & $\mathbf{2}$ & 232 & 1 & 2 & $\mathbf{0}$ & 31 & 32 \\
\hline
\end{tabular}

where each column represents $q$ (the current value of the state $q$ ), each row represents a value of $i$ (the wall that has been impacted by $z$ or $x$ ), and the number in each cell indicates $q^{+}$(the next value of the state $q$ ).

The construction of the automaton (8.299) is explained in the proof of the next property, which generalizes Properties 1 and 2 of the previous sections. Rather than 
giving a full and formal proof of this property, we explain it graphically with reference to Figure 8.39. An alternative mathematical proof can be constructed following the guidelines in [FOR 11c, Section V.G].

Property 3 For any diagonal positive definite matrix $P \in \Re^{2 \times 2}$, define $\bar{P}=P \otimes I_{2}$. Consider the hybrid closed loop (8.295), (8.296)-(8.298), (8.299). Then the jump map ensures that for all e in the jump set

$$
\left(e^{+}\right)^{T} \bar{P} e^{+} \leq e^{T} \bar{P} e .
$$

Proof. (Graphical sketch). Let us first consider jumps of the $z$ mass, namely lookup table in (8.299a) with reference to the mirrored images of Figure 8.39. First recall that $|e|_{P}=|x-m(q, z)|_{P}$ denotes the (generalized) distance from $x$ (which is the green mass in the central region where $q=0$ ) to the mirrored image of $z$ corresponding to $q$ (namely a specific column of the lookup table and a specific mirrored image of $z$ in the corresponding region of Figure 8.39). When an impact of $z$ occurs on wall $i \in\{1,2,3\}$, all the mirrored images impact on the edge corresponding to the mirror of the $i$-th wall, that is, the edge characterized by the small numbers $i$ next to it. Since the impact reverses the orthogonal velocity to the wall, the italicized update rules in the lookup table (8.299a) ensure that $q^{+}=\delta_{z}(q, i)$ characterizes the mirrored region on the opposite side of that edge. Therefore, $m\left(q^{+}, z^{+}\right)=m(q, z)$ and consequently $e^{+}=e$, which in turns implies $\left|e^{+}\right|_{\bar{P}}=|e|_{\bar{P}}$ as to be proven.

The bold numbers in (8.299a) characterize a different situation where the mirrored regions arising from the unfolding process of Figure 8.39 would move even beyond the set of white mirrors represented in the figure. Then it is possible to move back those regions closer to the central region (the actual billiard where $q=0$ ) due to the special structure of $\bar{P}$. Let us explain this in detail with reference to the three pink regions in Figure 8.39 (one at the top and two at the bottom) which are remapped to regions closer to the center as shown by the red arrows. Since $\bar{P}=P \otimes I_{2}$ with $P>0$ diagonal, then $|e|_{P}$ is the sum of four independent non-negative terms, two coming from the difference of the speed of $x$ and of the mirror $m(q, z)$ of $z$ and two coming from the position differences. Since each region is remapped to another mirror with the same orientation and the same horizontal position coordinate, then only the term that matters in the difference is the difference between the vertical displacements of $x$ and $m(q, z)$. Consider for example how $q=213$ and $q=1$ (lower left). Clearly, the vertical distance between $x$ and $m(1, z)$ is smaller than or equal to the triangle height $h$, while the vertical distance between $x$ and $m(123, z)$ is larger than or equal to $h$. Therefore, since the other three non-negative terms are the same, we get $\left|e^{+}\right|_{\bar{P}} \leq|e|_{\bar{P}}$. This motivates the bold $\mathbf{1}$ at the second row of the column "13" in the lookup table 
(8.299a). Similar reasonings motivate the other bold numbers corresponding to the gray triangles in Figure 8.39. Note that in many cases this inequality is indeed strict and a (negative) jump of the error can be expected.

Let us now focus on a jump of $x$ to explain the lookup table in (8.299b). Consider once again Figure 8.39 and notice that $x$ always belongs to the central region. Therefore, an impact of $x$ on the $i$-th wall, for some $i \in\{1,2,3\}$, corresponds to mirroring the velocity of $x$ with respect to this wall. The update of $q$ corresponding to the italicized numbers in $(8.299 \mathrm{~b})$ is then carried out to ensure that the mirrored image of $z$ jumps to the opposite side of the corresponding real wall, namely one among the orange (single lined), purple (double lined) or blue (triple lined) boundaries in Figure 8.39. Since both $x$ and $m(q, z)$ jump with respect to the same mirror, their distance does not change and the error remains constant. In particular, it can be shown that $\left|e^{+}\right|_{\bar{P}}=|\bar{M}(i)(x-m(q, z))|_{\bar{P}}=|x-m(q, z)|_{\bar{M}(i) \overline{P M}(i)}=$ $|x-m(q, z)|_{\bar{P}}$, where the last step comes from similar calculations to those carried out in (8.284). For illustration purposes, consider for example the case with $q=1$ and when $x$ impacts the second wall. Then Figure 8.39 suggests to mirror region " 1 " with respect to the purple double lined wall so to get $q^{+}=12$. This corresponds to the value at the second row of column " 1 " of table $(8.299 \mathrm{~b})$. The bold numbers in table ( $8.299 \mathrm{~b})$ can be explained similarly to the previous case. As an example, consider $q=12$ and impacting on the third wall, which would require mirroring the region " 12 " in Figure 8.39 into the pink region " 123 " at the bottom. Then, according to the bold $\mathbf{2}$ on the third row of column "12" of table (8.299b), we can select $q^{+}=\delta_{x}(12,3)=2$ and obtain $\left|e^{+}\right|_{\bar{P}} \leq|e|_{\bar{P}}$.

Using the result of Property 3 we can prove the exponential convergence to zero of the generalized error, as stated in the following theorem which parallels Theorems 48 and 49 of the previous sections.

Theorem 50 There exists $\gamma \geq 1$ and $\lambda>0$ such that every hybrid solution $\xi$ to the closed-loop system (8.295), (8.296)-(8.298), (8.299)) satisfies

$$
|e(t, j)| \leq \gamma \exp (-\lambda(t+j))|e(0,0)| \text {. }
$$

Similar to the previous sections, we need to ensure that the exponential bound on the generalized error indeed is associated to some kind of (weakened) convergence of $x$ to the actual mass $z$ and not to one of its mirrors. The following proposition parallels Propositions 16 and 17 of the previous sections. Its intuitive implication is clarified in Figure 8.39, indeed since all the mirrors are outside the region $q=0$ where the motion of $x$ is constrained to evolve, the only possibility for $x$ to flow with $e=0$ is that it coincides with the real target mass $z=m(0, z)$. This proof is sketched below. 
Proposition 18 Consider any solution $\xi$ to the hybrid closed-loop system (8.295), (8.296)-(8.298), (8.299)) starting from an initial condition satisfying $e(0,0)=0$. Then $e(t, j)=0$ for all $(t, j) \in \operatorname{dom}(e)$ and the solution cannot flow unless $x(t, j)=$ $z(t, j)$.

Proof. (Graphical sketch). Forward invariance of the set where $e=0$ comes from the exponential bound (8.301). Refer to Figure 8.39 for the rest of the proof. Let us focus on the considered case when $e(t, j)=0$, namely $x(t, j)=$ $m(q(t, j), z(t, j))$, and the solution flows. Since the position $x_{p}$ of $x=\left(x_{p}, x_{v}\right)$ belongs to $\mathcal{F}$ and the position of all the mirrors of $z$ with $q \neq 0$ do not belong to the interior of $\mathcal{F}$, the only possibility for the solution to flow with $e(t, j)=0$ and $q(t, j) \neq 0$ is that $x_{p}(t, j)$ is at a boundary of $\mathcal{F}$ and that $q(t, j) \in\{0,1,2,3\}$. On each boundary, the flow conditions (8.290b) and (8.291c) for $x$ and $z$ require that the speeds of $z$ and $x$ does not pierce the boundary. Then either $q(t, j)=0$ and both speeds point inwards (and coincide because $e(t, j)=0$ ) or $q(t, j) \in\{1,2,3\}$ and the speed vector are coincident and parallel to the boundaries (the mass slides along one boundary). In this latter case, we have $x(t, j)=z(t, j)$ even though $q(t, j) \neq 0$.

\subsubsection{Simulations}

We illustrate the proposed approach by simulating the trajectories of the hybrid closed-loop system starting from the initial conditions $z_{p}(0,0)=(-1,0.3)$, $z_{v}(0,0)=(2,2), x_{p}(0,0)=(-1.2,-0.5), x_{v}(0,0)=(0,0)$ for the triangle represented in Figure 8.40, which corresponds to:

$$
\left[F_{1}\left|F_{2}\right| F_{3}\right]=\left[\begin{array}{c|c|c}
0.7029 & -0.7029 & 0 \\
0.4058 & 0.4058 & -1
\end{array}\right] .
$$

Figure 8.40 shows three trajectories starting from different initial conditions $q(0,0)$, namely starting from tracking of different mirrors. The left picture of the figure illustrates the position trajectories of the two masses, while the right picture shows the evolution of the function $|e(t, j)|_{P}$ projected on the ordinary time direction $t$ for the three considered cases. In the left figure, the position of the real target is represented by the red dashed curve while the position of the controlled mass is represented in black when $q(0,0)=0$ (namely the tracking algorithm initially focuses on the real target mass $z$ ); it is represented in green when $q(0,0)=1$ (namely the tracking algorithm initially focuses on the target mass $m(1, z)$ mirrored through the upper left wall); it is represented in blue when $q(0,0)=3$ (namely the tracking algorithm initially focuses on the target mass $m(3, z)$ mirrored through the lower wall). Tracking performance and exponential decrease of the generalized error $e$ can be appreciated for any initial condition, as guaranteed by the results of Theorem 50 and Proposition 18. 

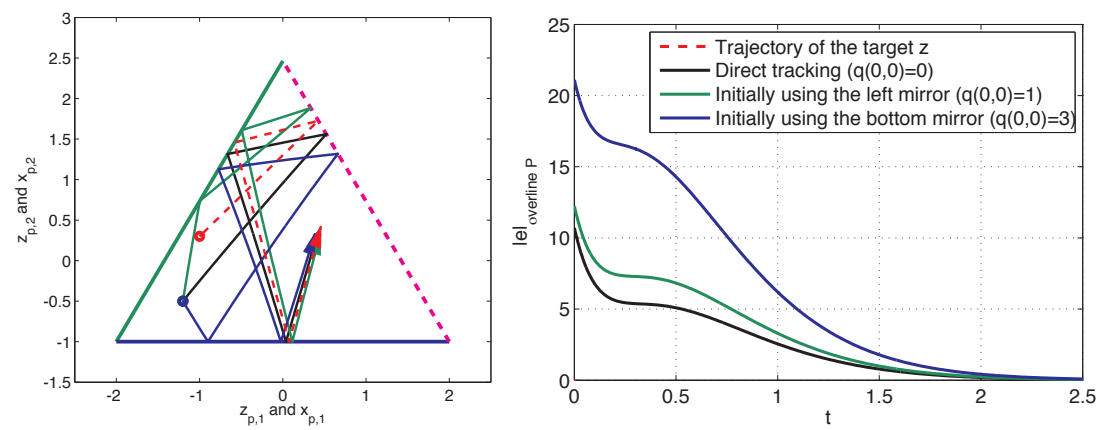

Figure 8.40: Trajectories of the hybrid tracking algorithm in a triangular region (left) and evolution of the generalized error (right).

\subsection{Global state estimation on $n$-dimensional convex polyhedra}

This section is apparently disconnected from the previous ones because it deals with state estimation rather than reference tracking and it also does not introduce any mirror to guarantee exponential convergence to zero of the error dynamics. Nevertheless, the underlying technique remains the same, as it will be clear from the proof of the main result of this section, and the absence of mirrors arises from the strong simplification (that one can only use with observer dynamics) of not constraining the motion of both $x$ and $z$ into the confined region $\mathcal{F}$.

\subsubsection{The reference dynamics}

We consider a state-estimation problem for a mass moving in an $n$-dimensional space, where we aim to make the observer state $x \in \Re^{2 n}$ asymptotically converge to the reference mass $z \in \Re^{2 n}$ whose position sub-vector belongs to a suitable polyhedron $\mathcal{F} \subset \Re^{n}$ defined by

$$
\mathcal{F}=\left\{s \in \Re^{n}: \forall i \in \mathcal{I},\left\langle F_{i}, s-s_{\circ}\right\rangle \leq 1\right\},
$$

with $F_{i} \in \Re^{n}, i \in \mathcal{I}=\{1, \ldots, r\} \subset \mathbb{N}$ denoting the vectors characterizing each wall of the $n$-dimensional polyhedral region $\mathcal{F}$ and $s_{\circ} \in \Re^{n}$ being a fixed point in the interior of the region. For example, if one moves $s_{\circ}$ while leaving $F_{i}, i \in \mathcal{I}$ unchanged, then the shape of the region $\mathcal{F}$ remains unchanged and its position is moved accordingly. The algorithm proposed below generalizes the hybrid observer in [MEN 01c], where the case of one boundary $I=\{1\}$ is considered in the planar case (that is, $n=2$ ). Moreover, parallel results for the tracking case and for the case of an $n$-dimensional space have been discussed in [FOR 12]. The approach proposed here is simpler than the one in [FOR 12] because for the tracking case the controlled system 
is forced to only lie in the region $\mathcal{F}$. Instead, here we allow the observer dynamics to flow even for positions outside $\mathcal{F}$ and this greatly simplifies the approach. An alternative approach where the observer is constrained to not flow outside $\mathcal{F}$ has been discussed in [FOR 11a] for the case $n=2$ and only for suitable polyhedral shapes. The core strategy presented here can be further generalized to convex sets $\mathcal{F}$ for which the normal vectors $F(s)$ to a point $s$ of the boundary $\partial \mathcal{F}$ are defined by a piecewise Lipschitz function $F: \partial \mathcal{F} \rightarrow \Re^{2}$. We do not follow this generality here for reasons of simplicity but the extension can be carried out in similar ways to the extension from the local results of [FOR 11b] to the local results of [FOR 11c].

Generalizing the approach of the previous section to an $n$-dimensional space, we consider a quasi-linear reference dynamics given by

$$
\dot{z}=\bar{A} z+\bar{B} \alpha\left(z_{p}\right) \quad z \in C_{z}:=\mathcal{K} \subset \mathcal{F} \times \Re^{n}
$$

where $\bar{A}:=\left[\begin{array}{ll}0 & 1 \\ 0 & 0\end{array}\right] \otimes I_{n}, \bar{B}:=\left[\begin{array}{l}0 \\ 1\end{array}\right] \otimes I_{n}$, and $\alpha\left(z_{p}\right) \in \Re^{n}$ is a known nonlinear term that depends only on the position sub-vector $z_{p}$. We assume that the closed set $\mathcal{K}$ restricts the motion of the reference away from the "corners" of the polyhedral region, i.e. the points $s_{p}$ such that $\left\langle F_{i}, s_{p}-s_{\circ}\right\rangle=\left\langle F_{j}, s_{p}-s_{\circ}\right\rangle=1$ for $i, j \in \mathcal{I}, i \neq j$. This assumption will avoid places where the impact dynamics may be a set valued map.

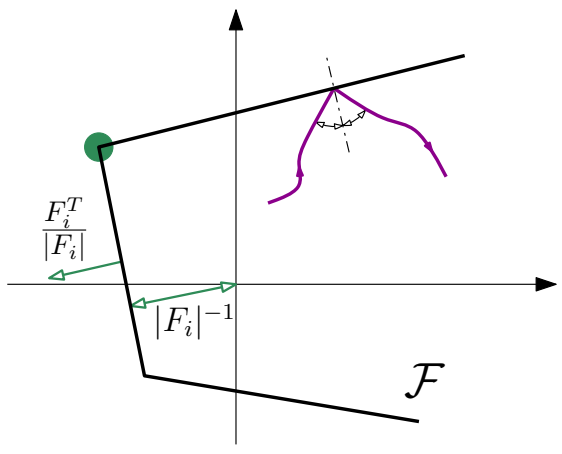

Figure 8.41: The polyhedral region $\mathcal{F}$ for the case $n=2$ with the representation of the effect of an impact.

Similar to the technique adopted in Section 8.4.1, the motion of the reference dynamics $z=\left(z_{p}, z_{v}\right)$ is restricted to the region $\mathcal{F} \times \Re^{n}$ by enforcing suitable impacts on the mass when $z_{p}$ belongs to the boundary $\partial \mathcal{F}$ of the polyhedron $\mathcal{F}$ and the velocity $z_{v}$ satisfies $\left\langle F_{i}, z_{v}\right\rangle \geq 0$, for some $i \in \mathcal{I}$. Once again, just as in (8.288), we use the formulation in [STR 03, page 220], which is equivalent to the one in [FOR 12] to 
describe an impact on the $i$ th wall as:

$$
M(i):=I_{n}-2 \frac{F_{i} F_{i}^{T}}{\left|F_{i}\right|^{2}},
$$

which inverts the component of $z_{v}$ orthogonal to $F_{i}$ (recall that $\left|F_{i}\right|=\sqrt{F_{i}^{T} F_{i}}$ ). We can then generalize the quantities introduced in (8.289) for the planar case as follows:

$$
\begin{aligned}
c(i) & :=F_{i} \cdot 2\left(1+F_{i}^{T} s_{\circ}\right) /\left|F_{i}\right|^{2} \\
\bar{M}(i) & :=I_{2} \otimes M(i) \\
\bar{c}(i) & :=\left[\begin{array}{ll}
c(i)^{T} & 0_{1 \times n}
\end{array}\right]^{T}=\left[\begin{array}{l}
1 \\
0
\end{array}\right] \otimes c(i) \\
m(i, z) & :=\bar{M}(i) z+\bar{c}(i) .
\end{aligned}
$$

Then, based on (8.304), (8.305), the impact dynamics coincides with that of the planar case in (8.290) and corresponds to

$$
z^{+}=m(i, z) \quad z \in D_{z}^{(i)}
$$

where for each $i \in \mathcal{I}$,

$$
\begin{aligned}
D_{z}^{(i)} & :=\left\{z \in \mathcal{K}:\left\langle F_{i}, z_{p}-s_{\circ}\right\rangle=1,\left\langle F_{i}, z_{v}\right\rangle \geq 0\right\} \\
D_{z} & :=\bigcup_{i \in \mathcal{I}} D_{z}^{(i)}=\partial \mathcal{F} \times \Re^{n} \cap \mathcal{K} .
\end{aligned}
$$

The reference dynamics formulation is then concluded by adding the average dwelltime logic (8.253) having state $\sigma$ and introduced in Section 8.2.2, to rule out Zeno solutions that occur when the reference mass impacts a wall $F_{i}$ with zero parallel component $z_{v}$ to $F_{i}$ (namely it slides along the wall). Similar to before, the parameters of the dwell time logic do not appear in the observer construction so that global results can be concluded.

\subsubsection{The observer dynamics}

Without impacts, following standard linear Luenberger constructions, we select the observer dynamics as a copy of the reference dynamics with a feedback injection term from the output error $x_{p}-z_{p}=\bar{H}(x-z)$, where $\bar{H}:=\left[\begin{array}{ll}1 & 0\end{array}\right] \otimes I_{n}$ :

$$
\begin{aligned}
\dot{x} & =\bar{A} x+\bar{B} \alpha\left(z_{p}\right)+\overline{L H} e \\
e & =x-z,
\end{aligned}
$$


where $\bar{L}=L \otimes I_{n}$ and $L=\left[\begin{array}{l}\ell_{1} \\ \ell_{2}\end{array}\right]$ is any output injection gain guaranteeing that the matrix $A_{c l}=\left[\begin{array}{ll}\ell_{1} & 1 \\ \ell_{2} & 0\end{array}\right]$ is Hurwitz. As in the previous section, the specific selection of $\bar{L}$ guarantees that $\bar{A}+\overline{L C}=A_{c l} \otimes I_{n}$ which is evidently a Hurwitz matrix because the eigenvalues of $X \otimes Y$ are given by the products of each pair of eigenvalues of $X$ and $Y$. Note also that $\bar{A}+\overline{L C}$ preserves the particular structure of the reference dynamics, which will be exploited in the hybrid reformulation of the observer algorithm.

Differently from the Newton's cradle, the observer is not affected by impact phenomena. Therefore, the design of the jump dynamics is an extra degree of freedom in the hybrid reformulation of the observer.

\subsubsection{Estimation by hybrid reformulation of the observer dynamics}

The hybrid reformulation of the observer is based on two main features: (i) the observer is forced to jump whenever the reference dynamics jumps; (ii) when the reference dynamics impacts the boundary of $\mathcal{F}$ on the $i$ th wall (namely it belongs to $\left.D_{z}^{(i)}\right)$, the observer state is reset to $x^{+}=m(i, x)$. Imposing this specific reset rule on the observer ensures the next property (we use properties from (8.305)):

$$
\begin{aligned}
e^{+} & =x^{+}=z^{+}=m(i, x)-m(i, z) \\
& =\bar{M}(i)(x-z)=\bar{M}(i) e .
\end{aligned}
$$

Property (8.308) is fundamental to ensure that at each impact of the reference mass the observer is suitably reinitialized to account for the state change caused by the impact. This was the core motivation behind the approach of [MEN 01c] and is extended here to a broader class of problems. Note also that the approach is substantially different from the Newton's cradle results of the previous section. Indeed there is no real need to constrain the motion of the observer in the region $\mathcal{F}$ and this greatly simplifies the analysis. More than that, the jump rule and jump set for the observer dynamics is an extra degree of freedom in the hybrid reformulation of the observer which is exploited to ensure the convenient linear behavior of $e$ across jumps characterized in (8.308).

Selecting the observer flow dynamics in (8.307), forcing the observer to jump synchronously with the reference dynamics and with the jump rule described above, one gets the following overall hybrid closed-loop system (also incorporating the average dwell-time logic):

$$
\begin{aligned}
& \left\{\begin{array}{l}
\dot{z}=\bar{A} z+\bar{B} \alpha\left(z_{p}\right) \\
\dot{x}=\bar{A} x+\overline{L C}(x-z)-\bar{B} \alpha\left(z_{p}\right) \quad(z, x, \sigma) \in C_{z} \times \Re^{4} \times[0, N] . \\
\dot{\sigma} \in[0, \rho]
\end{array}\right. \\
& \left\{\begin{array}{ll}
z^{+}=m(i, z) \\
x^{+}=m(i, x) \\
\sigma=
\end{array} \quad(z, x, \sigma) \in D_{z}^{(i)} \times \Re^{4} \times[1, N],\right.
\end{aligned}
$$


for all $i \in \mathcal{I}$. The hybrid dynamics (8.309), (8.310) characterizes a hybrid closed-loop system having flow set $C=C_{z} \times \Re^{4} \times[0, N]$ and jump set $\bigcup_{i \in \mathcal{I}} D_{z}^{(i)} \times \Re^{4} \times[1, N]$ where, due to the restriction of the $z$ motion to the compact set $\mathcal{K}$, the union is carried out among disjoint sets (in other words, no impacts on corners occur).

The hybrid observer proposed above is a straightforward hybrid extension of a continuous-time Luenberger observer, based on a suitable definition of the jump dynamics. In particular, in comparison to the hybrid controller for the tracking of the Newton's cradle, there is no need to introduce any mirrored reference or any generalized error. This is a consequence of exploiting extra degrees of freedom for the continuous dynamics of the observer, whose position sub-vector is not constrained to lie in the polyhedral region $\mathcal{F}$ during transients. The generalized error may be introduced to formulate alternative observation laws where this constraint is enforced, as carried out in [FOR 11a] and [FOR 12]. Nevertheless the analysis in those cases is more involved and does not allow to achieve global results for a large class of polyhedral regions (which we obtain here).

Despite the absence of mirrored references, the rationale behind the stability analysis that we carry out here is parallel to the one adopted in Section 8.3 and corresponds to exploiting the following property, which evidently parallels Property 2.

Property 4 For any matrix $P \in \Re^{2 \times 2}$, define $\bar{P}=P \otimes I_{n}$. Consider the hybrid closed loop (8.309)-(8.310) and the error variable $e=x-z$ in (8.307). Then, for all $e$ in the jump set of (8.309)-(8.310), we have

$$
\left(e^{+}\right)^{T} \bar{P} e^{+}=e^{T} \bar{P} e .
$$

Proof. The proof relies upon (8.308) and similar calculations to those of (8.284) in the proof of Property 2. Indeed, from the definition of $\bar{M}(i)$ in (8.305b) and from the structure of $\bar{P}$, we can use the properties of the Kronecker product to conclude that for each $i \in \mathcal{I}, \bar{M}(i) \overline{P M}(i)=\bar{P}$ (recall also that $\bar{M}(i) \bar{M}(i)=I$ for all $i \in \mathcal{I}$ as shown in [STR 03, page 220]). Then the result follows by combining the previous inequality with the identity in (8.308).

Similar to the previous sections, the unifying Lyapunov result given in Section 8.6.1 combined with Property 4 allows us to state the following result establishing global exponential stability of the error dynamics. Its proof is given in Section 8.6.2. 
Theorem 51 There exists $\gamma>1$ and $\lambda>0$ such that every hybrid solution $\xi$ to the closed-loop system (8.309),(8.310) satisfies

$$
|e(t, j)| \leq \gamma \exp (-\lambda(t+j))|e(0,0)|,
$$

where $e=x-z$ as defined in (8.307).

Note that since in the case study addressed in this section we did not introduce any mirrored reference, there is no need of proving a parallel result to that of Proposition 17. Indeed the conclusion of Theorem 51 implies that for any solution of the hybrid closed-loop system we have an exponential bound on $e(t, j)=x(t, j)-z(t, j)$ which implies exponential convergence of the estimate $x$ to the state $z$ despite the impacts occurrence.

\subsubsection{Simulations}

We illustrate the proposed approach on a planar example (namely $n=2$ ) where the polyhedron is a rectangle described by $s_{\circ}=0$ and

$$
\left[F_{1}\left|F_{2}\right| F_{3} \mid F_{4}\right]:=\frac{1}{4}\left[\begin{array}{l|l|l|c}
3 & 0 & -3 & 0 \\
0 & 4 & -0 & -4
\end{array}\right] .
$$

We also consider a reference dynamics comprising a pair of double integrators, so that $\alpha\left(z_{p}\right)=0$ for all $z_{p}$.

Figure 8.42 shows the simulation results when the observer gain is selected as $L=\left[\begin{array}{l}-4 \\ -4\end{array}\right]$, so that the eigenvalues of $A_{c l}$ are both in -2 . The initial conditions of the reference and observer dynamics is selected as $z_{0}=\left[\begin{array}{llll}0.1 & 0.1 & 15 & 7\end{array}\right]^{T}$ and $x_{0}=$ $\left[\begin{array}{llll}-0.5 & 0.1 & -10 & -3\end{array}\right]^{T}$. The upper two plots of the figure, representing the horizontal and vertical positions projected in the $t$ axis, respectively, show that the observer state (black trace) takes position values well outside the region $\mathcal{F}$ where the motion of the reference mass is constrained. This degree of freedom within the (virtual) observer dynamics is a key tool for establishing global results for such a general class of systems. Alternative observation laws which exploit the mirroring technique of this chapter and constrain the motion of the observer state within the region $\mathcal{F}$ have also been proposed in recent years, but global results have only been proven for planar regions with special shapes, including rectangles. As an illustration of this, an observer with constrained motion has been given for this same example in [FOR 11a].

\subsection{Proof of the main theorems}

\subsubsection{A useful Lyapunov result}

In this section we introduce a Lyapunov result which establishes an exponential stability property that we will use for each of the proposed examples. This result, 

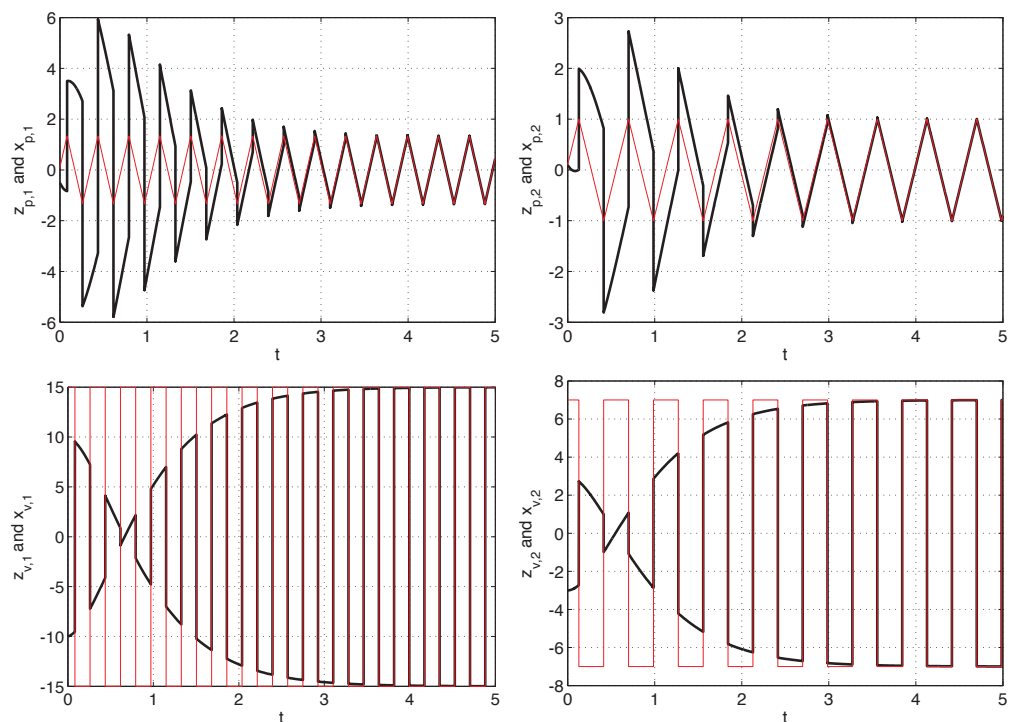

Figure 8.42: Simulation of the hybrid observer on a planar example. The upper graphs show the positions while the lower graphs show the velocity. Reference:red, observer: black.

suitably combined with the properties established in each one of the studied examples, will allow to prove the exponential properties stated in Theorems 48, 49, 51 which are yet to be proven. Each one of the above theorems will be proven in the next section based on the following lemma.

Lemma 12 Consider a hybrid dynamical system having state $(e, \xi)$ and flow and jump sets $C_{\xi}, D_{\xi}$, respectively. Assume that all solutions to this hybrid system satisfy an average dwell-time condition, that the flow dynamics restricted to e corresponds to the following linear relation:

$$
\dot{e}=\bar{A}_{c l} e, \quad(e, \xi) \in C_{\xi} .
$$

Assume also that there exists a positive definite matrix $\bar{P}$ and a matrix $\bar{H}$ such that $\left(\bar{H}, \bar{A}_{c l}\right)$ is an observable pair and

$$
\begin{array}{llrl}
e^{T}\left(\overline{P A}_{c l}\right) e & \leq-e^{T} \bar{H}^{T} \bar{H} e, & \forall(e, \xi) & \in C_{\xi}, \\
\left(e^{+}\right)^{T} \bar{P} e^{+} \leq e^{T} \bar{P} e, & \forall(e, \xi) \in D_{\xi} .
\end{array}
$$


Then there exists $\gamma \geq 1$ and $\lambda>0$ such that all solutions to the hybrid dynamical system satisfy

$$
|e(t, j)| \leq \gamma \exp (-\lambda(t+j))|e(0,0)| .
$$

Proof. The result is a direct consequence of [TEE 12, Theorem 2]. In particular, [TEE 12, Assumption 1] holds under the conditions of the lemma with $x_{1}=e$, $x_{2}=\xi, F_{1}=\bar{A}_{c l}, H_{1}=\bar{H}, V(x)=V((e, \xi))=e^{T} \bar{P} e$ and $\mu=\infty$. Then, using the notation of [TEE 12, Theorem 2], we have $|x|_{\mathcal{A}}=|e|$ and the global exponential stability of $\mathcal{A}$ established by [TEE 12, Theorem 2] corresponds to the exponential bound (8.317).

\subsubsection{Proofs of Theorems 48, 49, 50 and 51}

In this section we prove the main theorems stated in each one of the examples addressed in this chapter. The proof of each statement will rely heavily on the Lyapunov result of Lemma 12. The motivation for grouping these proofs in a single section is to highlight the unified nature of the approach illustrated in this chapter which provides a different viewpoint on the result of our recent works [FOR 11b, FOR 11c, FOR 11a, FOR 12].

For the proofs of Theorems 48, 49, 50 and 51 below, we will first show that the generalized error dynamics satisfies (8.314) for a suitable Hurwitz matrix $\bar{A}_{c l}$. Then a positive definite symmetric matrix $\bar{P}$ will be constructed to satisfy $(8.315)$ and at the same time respect the structure required, respectively, in Properties 1, 2, 3, and 4. Indeed, each one of these properties ensures (in different ways) that equation (8.316). So that finally Lemma 12 can be applied to prove the corresponding theorem. The most relevant difference among the various examples probably resides on the corresponding property. In particular, Property 1 ensures that $e^{T} \bar{P} e$ remains constant at each jump for any symmetric positive definite $\bar{P}$, while Property 2 concludes the same result as long as $\bar{P}=P \otimes I_{2}$. With Property 3 things are much more difficult because $\bar{P}$ must be diagonal and even with such a constrained structure, one gets nonincrease of $e^{T} \bar{P} e$ even though there might be cases when the quantity experiences a finite nonzero decrease. The diagonal structure of $\bar{P}$ also does not allow to obtain a "strict" decrease in (8.315) but the conditions of Lemma 12 are weak enough to allow for a non-full rank $\bar{H}$ in (8.315) as long as $\left(\bar{H}, \bar{A}_{c l}\right)$ is an observable pair. Finally, Property 4 resembles the case of Property 2 with the similar structure $\bar{P}=P \otimes I_{n}$ for $\bar{P}$.

Proof of Theorem 48. We prove the theorem by showing that all the assumptions of Lemma 12 hold for the hybrid closed-loop system (8.256)-(8.258), (8.262)-(8.265). 
Consider first the flow dynamics (8.256) and note that

$$
\begin{aligned}
\dot{e} & =\dot{x}-q \dot{z} \\
& =\bar{A} x+\bar{B}\left(\bar{K} e+q \alpha_{z}(z)\right)-q\left(\bar{A} z+\bar{B} \alpha_{z}(z)\right) \\
& =\bar{A} x-q \bar{A} z+\overline{B K}=\bar{A}_{c l} e,
\end{aligned}
$$

where $\bar{A}_{c l}$ is Hurwitz by the construction of $\bar{K}$. So considering $\xi=(z, q, \sigma)$, the hybrid closed-loop system (8.256)-(8.258), (8.262)-(8.265) (in the transformed coordinates $(e, z, q, \sigma)$ satisfies equation (8.314) of Lemma 12.

Consider now any positive definite matrix $\bar{Q} \in \Re^{2 \times 2}$ and the symmetric positive definite solution $\bar{P}$ to the (always feasible) Lyapunov equation $\bar{A}_{c l}^{T} \bar{P}+\overline{P A}_{c l}=-\bar{Q}$. Then performing the Cholesky decomposition of $\bar{Q}=\bar{H}^{T} \bar{H}$, condition (8.315) in Lemma 12 holds and $\left(\bar{H}, \bar{A}_{c l}\right)$ is observable because $\bar{H}$ is full rank. Finally relations (8.266) established in Property 1 imply that the quadratic left-hand side and right-hand side of (8.316) always coincide and Lemma 12 can be applied to complete the proof.

Proof of Theorem 49. First define, as in the proof of Property 2, $\tilde{M}(0)=I, \tilde{M}(1)=$ $M$, and $\widetilde{\widetilde{M}}(q)=I_{2} \otimes \tilde{M}(q)$ for $q \in\{0,1\}$. Then we have $m(q, z)=\widetilde{\bar{M}}(q) z$. Now, using the definition of $\bar{B}$ in (8.272) and the properties of the Kronecker products, we have:

$$
\begin{aligned}
\widetilde{\bar{M}}(q) \bar{B} & =\left(I_{2} \otimes \tilde{M}(q)\right)\left(\left[\begin{array}{l}
0 \\
1
\end{array}\right] \otimes I_{2}\right) \\
& =\left[\begin{array}{l}
0 \\
1
\end{array}\right] \otimes \tilde{M}(q)=\left(\left[\begin{array}{l}
0 \\
1
\end{array}\right] 1\right) \otimes\left(I_{2} \tilde{M}(q)\right) \\
& =\left(\left[\begin{array}{l}
0 \\
1
\end{array}\right] \otimes I_{2}\right)(1 \otimes \tilde{M}(q))=\bar{B} \tilde{M}(q) \\
\bar{B}^{T} \widetilde{\bar{M}}(q) \bar{B} & =\left(\left[\begin{array}{ll}
1 & 1
\end{array}\right] \otimes I_{2}\right)\left(I_{2} \otimes \tilde{M}(q)\right)\left(\left[\begin{array}{l}
0 \\
1
\end{array}\right] \otimes I_{2}\right) \\
& =\left(\left[\begin{array}{ll}
0 & 1
\end{array}\right] \otimes I_{2}\right)\left(\left[\begin{array}{l}
0 \\
1
\end{array}\right] \otimes \tilde{M}(q)\right) \\
& =1 \otimes \tilde{M}(q)=\tilde{M}(q) .
\end{aligned}
$$

Consider now the error variable $e$ defined in (8.279). Using equations (8.279), (8.280) and identities (8.319), we can compute its time derivative along flows, corresponding to

$$
\begin{aligned}
\dot{e} & =\dot{x}-\widetilde{\bar{M}}(q) \dot{z} \\
& =\bar{A} x+\bar{B}\left(\bar{K} e+\bar{B}^{T} m(q, \bar{B} \alpha(z))\right)-\widetilde{\bar{M}}(q)(\bar{A} z+\bar{B} \alpha(z)) \\
& =\bar{A} e+\bar{B}\left(\bar{K} e+\bar{B}^{T} \widetilde{\bar{M}}(q) \bar{B} \alpha(z)-\tilde{M}(q) \alpha(z)\right) \\
& =(\bar{A}+\overline{B K}) e=\bar{A}_{c l} e,
\end{aligned}
$$


where $\bar{A}_{c l}=\left(\left[\begin{array}{ll}0 & 1 \\ 0 & 0\end{array}\right]+\left[\begin{array}{l}0 \\ 1\end{array}\right] K\right) \otimes I_{2}=A_{c l} \otimes I_{2}$ is Hurwitz because of the construction of $K$. So considering $\xi=(z, q, \sigma)$, the hybrid closed-loop system (8.279)-(8.282) (in the transformed coordinates $(e, z, q, \sigma)$ satisfies equation (8.314) of Lemma 12.

Consider now any positive definite matrix $P=P^{T} \in \Re^{2 \times 2}$ such that $A_{c l}^{T} P+$ $P A_{c l}=-Q<0$ and select $\bar{P}=P \otimes I_{2}, \bar{H}$ based on the Cholesky decomposition of the positive definite symmetric matrix $Q \otimes I_{2}=\bar{H}^{T} \bar{H}$. Then condition (8.315) in Lemma 12 holds from the properties of the Kronecker product. Moreover, since $\bar{P}$ has the structure required in Property 2 , condition (8.316) holds too. Observability of $\left(\bar{H}, \bar{A}_{c l}\right)$ trivially follows from non-singularity of $\bar{H}$. Finally, the bound (8.317) obtained from Lemma 12 completes the proof of Theorem 49.

Proof of Theorem 50. Proceeding in similar ways to the previous proof, first notice that based on the definitions in (8.289), (8.294) for each $q \in \mathcal{Q}$, we can define $M(q)$ and $c(q)$ similar to (8.289) so that

$$
m(q, z)=\bar{M}(q) z+\bar{c}(q)=\left(I_{2} \otimes M(q)\right) z+\left(\left[\begin{array}{l}
1 \\
0
\end{array}\right] \otimes c(q)\right) .
$$

Then similar calculations to those in (8.319) provide

$$
\begin{aligned}
\bar{M}(q) \bar{B} & =\bar{B} M(q), \\
\bar{B}^{T} \bar{M}(q) \bar{B} & =M(q) .
\end{aligned}
$$

Based on identities (8.321), a similar relation to (8.320) can be determined using for the generalized error flow dynamics arising from (8.295), (8.296):

$$
\begin{aligned}
\dot{e} & =\dot{x}-\bar{M}(q) \dot{z} \\
& =\bar{A} x+\bar{B}\left(\bar{K} e+\bar{B}^{T} m(q, \bar{B} \alpha(z))\right)-\bar{M}(q)(\bar{A} z+\bar{B} \alpha(z)) \\
& =\bar{A} e+\bar{B}\left(\bar{K} e+\bar{B}^{T} \bar{M}(q) \bar{B} \alpha(z)-M(q) \alpha(z)\right) \\
& =(\bar{A}+\overline{B K}) e=\bar{A}_{c l} e,
\end{aligned}
$$

where $\bar{A}_{c l}=\left(\left[\begin{array}{ll}0 & 1 \\ 0 & 0\end{array}\right]+\left[\begin{array}{l}0 \\ 1\end{array}\right] K\right) \otimes I_{2}=A_{c l} \otimes I_{2}$ is Hurwitz because of the construction of $K$. So considering $\xi=(z, q, \sigma)$, the hybrid closed-loop system (8.295), (8.296)-(8.298) (in the transformed coordinates $(e, z, q, \sigma)$ satisfies equation (8.314) of Lemma 12.

The proof is completed similarly to the proof of Theorem 49, with the exception that $P=P^{T}>0$ should be selected diagonal so that Property 3 can be applied. Note that since the matrix $A_{c l}$ is in observer canonical form, then with a diagonal $P$ and with $\bar{P}=P \otimes I_{2}$, one gets $e^{T}\left(\overline{P A}_{c l}\right) e=-e^{T} \bar{H}^{T} \bar{H} e$ where $\bar{H}=H \otimes I_{2},\left(H, A_{c l}\right)$ is observable because $A_{c l}$ is in observer canonical form and, consequently, $\left(\bar{H}, \bar{A}_{c l}\right)$ is observable too. 
Proof of Theorem 51. As compared to the previous proofs, the hybrid closed loop (8.309)-(8.310) presents an easier analysis because there is no variable $q$ and, according to (8.307), we have $e=x-z$. Then, using the flow dynamics (8.309) we have (8.295), (8.296):

$$
\dot{e}=\dot{x}-\dot{z}=(\bar{A}+\overline{L C})(x-z)=\bar{A}_{c l} e,
$$

where $\bar{A}_{c l}=A_{c l} \otimes I_{n}$ is Hurwitz due to similar reasonings as those in the previous proofs. Then the hybrid closed loop (8.309)-(8.310) (in the transformed coordinates $(e, z, \sigma)$ satisfies equation (8.314) of Lemma 12.

Since Property 4 is analogous to Property 2 used in the proof of Theorem 49, above, the proof is completed following exactly the same steps as in the end of the proof of Theorem 49 .

\subsection{Conclusions}

In this chapter a number of example studies have been proposed to illustrate the suggestive idea of addressing tracking and observation problems for continuous-time dynamical systems experiencing impacts by way of introducing suitable reflections of the reference motion by way of some mirrors constructed based on the impacting surfaces. This mirroring technique allows defining a generalized error coordinates whose dynamical behavior is well characterized within a suitable hybrid formulation of the overall dynamics. For each one of the proposed examples, the analysis behind exponential convergence to zero of the error dynamics has been illustrated in an intuitive way in this chapter. To better emphasize the commonalities among all the apparently different example studies, the proofs of the main results have been gathered together in the last section where differences and similarities can be best appreciated.

Acknowledgements Fulvio Forni: this paper presents research results of the Belgian Network DYSCO (Dynamical Systems, Control, and Optimization), funded by the Interuniversity Attraction Poles Programme, initiated by the Belgian State, Science Policy Office; the scientific responsibility rests with its author(s). Andrew R. Teel: research supported in part by the National Science Foundation under grant ECCS1232035 and by the Air Force Office of Scientific Research under grant FA955012-1-0127. Luca Zaccarian: work supported by HYCON2 Network of Excellence "Highly-Complex and Networked Control Systems", grant agreement 257462.

\subsection{Bibliography}

[BIE 12] Biemond J., van de Wouw N., Heemels W., NiJmeijer H., "Tracking control of mechanical systems with impacts", IEEE American Control Conference, Montreal, Canada, p. 258-263, June 2012. 
[BIE 13] Biemond J., VAn de Wouw N., Heemels W., NiJMeiJer H., "Tracking Control for Hybrid Systems with State-Triggered Jumps”, IEEE Trans. Aut. Cont., to appear, 2013.

[BOU 05] Bourgeot J., BRogliato B., "Tracking control of complementarity Lagrangian systems", Int. J. Bifurcation Chaos, vol. 15, num. 6, p. 1839-1866, 2005.

[BRO 97] Brogliato B., Niculescu S. I., ORthant P., "On the control of finitedimensional mechanical systems with unilateral constraints", IEEE Transactions on Automatic Control, vol. 42, num. 2, p. 200-215, 1997.

[BRO 99] Brogliato B., Nonsmooth mechanics: models, dynamics, and control, Springer Verlag, 1999.

[BRO 04] BROGLIATO B., "Absolute stability and the Lagrange-Dirichlet theorem with monotone multivalued mappings", Systems \& control letters, vol. 51, num. 5, p. 343-353, 2004.

[CAI 08] Cai C., Teel A., Goebel R., "Smooth Lyapunov functions for hybrid systems Part II:(pre) asymptotically stable compact sets", IEEE Transactions on Automatic Control, vol. 53, num. 3, p. 734-748, 2008.

[FOR 11a] Forni F., TEel A., ZaCCARIAN L., "Follow the bouncing ball: global results on tracking and state estimation with impacts", IEEE Trans. Aut. Cont., submitted, 2011.

[FOR 11b] FORNI F., TEEL A., ZACCARIAN L., "Tracking control in billiards using mirrors without smoke, Part I: Lyapunov-based local tracking in polyhedral regions", Joint CDCECC, Orlando (FL), USA, p. 3283-3288, December 2011.

[FOR 11c] Forni F., TeEl A., ZaCCARIAN L., "Tracking control in billiards using mirrors without smoke, Part II: additional Lyapunov-based local and global results", Joint CDCECC, Orlando (FL), USA, p. 3289-3294, December 2011.

[FOR 12] Forni F., TeEl A., ZaCCARIAN L., "Global tracking and state estimation with nonsmooth impacts for a mass confined to a half $n$-dimensional space", 4th IFAC Conference on Analysis and Design of Hybrid Systems (ADHS), Eindhoven, The Netherlands, p. 376-381, June 2012.

[Gal 03] Galeani S., Menini L., Tornambe A., "A local observer for linearly observable nonlinear mechanical systems subject to impacts", Proceedings of the 2003 American Control Conference., vol. 6, p. 4760 - 4765 vol.6, June 2003.

[Gal 08] Galeani S., Menini L., Potini A., Tornambè A., "Trajectory tracking for a particle in elliptical billiards", Int. J. of Control, vol. 81, num. 2, p. 189-213, February 2008.

[GOE 06] Goebel R., Teel A., "Solutions to hybrid inclusions via set and graphical convergence with stability theory applications", Automatica, vol. 42, num. 4, p. 573 - 587, 2006.

[GOE 09] Goebel R., Sanfelice R., Teel A., "Hybrid dynamical systems", Control Systems Magazine, IEEE, vol. 29, num. 2, p. 28-93, April 2009.

[GOE 12] Goebel R., Sanfelice R., Teel A., Hybrid Dynamical Systems: modeling, stability, and robustness, Princeton University Press, 2012.

[HEE 03] Heemels W., BRogliato B., "The complementarity class of hybrid dynamical systems", European Journal of Control, vol. 9, num. 2-3, p. 322-360, 2003. 
[LEI 08a] LEINE R., VAN DE WOUw N., Stability and convergence of mechanical systems with unilateral constraints, Lecture Notes in Applied and Computational Mechanics, Springer Verlag, 2008.

[LEI 08b] LEINE R., VAN DE WOUW N., "Uniform convergence of monotone measure differential inclusions: with application to the control of mechanical systems with unilateral constraints", International Journal of Bifurcation and Chaos in Applied Sciences and Engineering, vol. 18, num. 5, p. 1435-1457, 2008.

[MEN 01a] Menini L., Tornambè A., "Asymptotic Tracking of Periodic Trajectories for a Simple Mechanical System Subject to Nonsmooth Impacts", IEEE Trans. Aut. Control, vol. 46, p. 1122-1126, 2001.

[MEN 01b] Menini L., ToRnambè A., "Dynamic position feedback stabilization of multidegrees-of-freedom linear mechanical systems subject to non-smooth impacts", IEE Proceedings of Control Theory and Applications, vol. 148, num. 6, p. 488-496, November 2001.

[MEN 01c] Menini L., ToRnambè A., "Velocity observers for linear mechanical systems subject to single non-smooth impacts", Systems \& Control Letters, vol. 43, p. 193-202, 2001.

[MEN 02] Menini L., Tornambe A., "An observer for the Newton's cradle: a nonlinear mechanism unobservable in the absence of impacts", Proceedings of the 41st IEEE Conference on Decision and Control, vol. 1, p. 682 - 683 vol.1, dec. 2002.

[MOR 09] MORRIS B., GRIZzLE J., "Hybrid invariant manifolds in systems with impulse effects with application to periodic locomotion in bipedal robots", IEEE Trans. Aut. Cont., vol. 54, num. 8, p. 1751-1764, 2009.

[MOR 10] Morarescu I., Brogliato B., "Trajectory tracking control of multiconstraint complementarity Lagrangian systems", IEEE Transactions on Automatic Control, vol. 55, num. 6, p. 1300-1313, 2010.

[NEĨ1] NeŠIĆ D., TeEl A., ZACCARIAn L., "Stability and performance of SISO control systems with First Order Reset Elements", IEEE Trans. Aut. Cont., vol. 56, num. 11, p. $2567-$ 2582, 2011.

[PAG 01] Pagilla P., "Control of contact problem in constrained Euler-Lagrange systems", IEEE Trans. Aut. Cont., vol. 46, num. 10, p. 1595-1599, 2001.

[PAG 04] PAGILla P., YU B., "An experimental study of planar impact of a robot manipulator", IEEE/ASME Transactions on Mechatronics, vol. 9, num. 1, p. 123-128, 2004.

[RON 06] Ronsse R., LeFÈvre P., SEPUlchre R., "Sensorless stabilization of bounce juggling”, IEEE Trans. on Robotics, vol. 22, num. 1, p. 147-159, 2006.

[SCH 95] Schwind W., KoditscheK D., "Control of forward velocity for a simplified planar hopping robot", IEEE International Conference on Robotics and Automation, vol. 1, p. 691-696, 1995

[SEK 06] Sekhavat P., SePehri N., Wu Q., "Impact stabilizing controller for hydraulic actuators with friction: Theory and experiments", Control Engineering Practice, vol. 14, num. 12, p. 1423-1433, 2006 
[STR 03] STRANG G., Introduction to linear algebra, 3rd edition, Wellesley Cambridge Press, 2003.

[TEE 12] TEel A., Forni F., ZaCCARIAn L., "Lyapunov-based sufficient conditions for exponential stability in hybrid systems", IEEE Trans. Aut. Cont., to appear, 2012.

[TOR 99] TORnAmbE A., "Modeling and control of impact in mechanical systems: Theory and experimental results", IEEE Trans. Aut. Cont., vol. 44, num. 2, p. 294-309, 1999. 